\title{
Linear and Sublinear Time Algorithms for the Basis of Abelian Groups
}

\author{
Li Chen*and Bin $\mathrm{Fu}^{\dagger}$
}

\begin{abstract}
It is well known that every finite Abelian group $G$ can be represented as a direct product of cyclic groups: $G=G_{1} \circ G_{2} \circ \cdots \circ G_{t}$, where each $G_{i}$ is a cyclic group of size $p^{j}$ for some prime $p$ and integer $j \geq 1$. If $a_{i}$ is the generator of the cyclic group of $G_{i}, i=1,2, \cdots, t$, then the elements $a_{1}, a_{2}, \cdots, a_{t}$ are called a basis of $G$. In this paper, we first obtain an $O(n)$-time deterministic algorithm for computing the basis of an Abelian group with $n$ elements. The existing algorithms need $O\left(n^{2}\right)$ time by Chen and $O\left(n^{1.5}\right)$ time by Buchmann and Schmidt. We then derive an $O\left(\left(\sum_{i=1}^{k} p_{i}^{n_{i} / 2} n_{i}^{2}\right)(\log n) \log \log n\right)$-time randomized algorithm to compute the basis of Abelian group $G$ of size $n$ with factorization $n=p_{1}^{n_{1}} \cdots p_{t}^{n_{t}}$, which is also a part of the input. It implies an $O\left(n^{1 / 2}(\log n)^{3} \log \log n\right)$-time randomized algorithm to compute the basis of an Abelian group $G$ of size $n$. It also implies that if $n$ is an integer in $\{1,2, \cdots, m\}-G(m, c)$, then the basis of an Abelian group of size $n$ can be computed in $O\left((\log n)^{\frac{c}{2}+3} \log \log n\right)$-time, where $c$ is any positive constant and $G(m, c)$ is a subset of the small fraction of integers in $\{1,2, \cdots, m\}$ with $\frac{|G(m, c)|}{m}=O\left(\frac{1}{(\log m)^{c / 2}}\right)$ for every integer $m$.
\end{abstract}

(Key words: Basis of Abelian Group; Linear time; Sublinear Time; Randomization)

\section{Introduction}

The theory of groups is a fundamental theory of mathematics. Its applications can be found throughout entire mathematics and theoretical physics especially quantum mechanics. In recent years, interest in studying the computational complexity of groups has raised dramatically due to the ever-increasing significance of its relationship to quantum computing and its application in elliptic curve cryptography. Since the early developmental period of computational complexity, computer scientists have shown great interest in the study of groups.

Abelian groups are groups with commutative property. It is well known that a finite Abelian group can be decomposed to a direct product of cyclic groups with prime-power order (called cyclic p-groups) [10]. This fundamental theorem is also called the Kronecker decomposition theorem. In quantum computing, the hidden subgroup problem (HSP) greatly interests scientists. The finite Abelian case was first used to spectacular effect by Shor and Simon [22,23]. "If the group G is Abelian, then it is possible to solve the HSP in polynomial time with bounded error on a quantum computer." A polynomial time algorithm in quantum computing means an algorithm whose running time is a polynomial of the logarithm of the size of the group. There is a polynomial time quantum algorithm for solving HSP over Abelian groups [4, 6, 13, 15, 16, 19, 22, 23]. The famous Shor's quantum algorithm to factorize integers is one special case.

On the other hand, finite Abelian groups have been used in elliptic curve cryptosystems that were introduced by Miller in 1986 [19]. This system is based on the discrete logarithm problem, which is as follows: given an element $g$ in a finite group $G$ (over an elliptic curve) and another element $h$ in $G$, find an integer $x$ such that $g^{x}=h[16]$. The advantage of using elliptic curve crypto-systems over other public-key crypto-systems is that the elliptic curve system may lead to smaller key sizes and better performance with the similar level of security. Another application in cryptography can be found in [6]

*Address: Department of Computer Science, University of District of Columbia, Washington, DC 20008, USA, Email:Ichen@udc.edu. Phone: 202-274-6301.

${ }^{\dagger}$ Address: Department of Computer Science, University of Texas-Pan American, Edinburg, TX 78539, USA, Email: binfu@cs.panam.edu. Phone: 956-381-3635. Fax: 956-384-5099. 
No polynomial-time algorithm has been found for determining if two general groups are isomorphic. The group isomorphism problem is related to the graph isomorphism problem and is also easier to solve than the graph isomorphism problem [18]. Hoffmann published a book in 1982 that presents interesting algebraic results that relate the graph isomorphism problem to the automorphism groups of the two graphs [8]. The related development can be found in [14]. Miller [17] showed an $O\left(n^{\log n+O(1)}\right)$ time algorithm for the group isomorphism problem. Savage [20] claimed the isomorphism between two Abelian groups can be checked in $O\left(n^{2}\right)$ steps. Vikas [24] improved it to $O(n)$ time for the Abelian $p$-group and $O(n \log n)$ time for Abelian group. Kavitha [12] showed that the Abelian group isomorphism problem can be computed in $O(n \log \log n)$ time. Garzon and Zalcstein [7] also discussed that the polynomial time algorithms for the isomorphism problem of Abelian groups. Recently, Kavitha [11], using a new method for computing the orders of all elements in $O(n)$ time, obtained $O(n)$ time algorithm for the Abelian group isomorphism problem. The methods for computing the order for one element in an Abelian group was also reported in [1,21]. In [2], Buchmann and Schmidt showed an $O(m \sqrt{|G|})$ time algorithm to compute the basis of an Abelian group $G$ with a set of generator of size $m$. Their result implies an $O\left(n^{1.5}\right)$ time algorithm for computing the basis of an Abelian group of size $n$. It is easy to see that an algorithm for finding the basis of Abelian group can be easily converted to the algorithm of checking the isomorphism of two Abelian groups.

Since the basis of an Abelian group fully determines its structure, finding the basis is crucial in computing the general properties for Abelian groups. The orders of all elements in a basis form the invariant structure of an Abelian group. Two Abelian group are isomorphic if and only if they have the same structure, which can be determined by the orders of elements in basis. Also, finding the basis of an Abelian group is the generalization of the integer factorization problem, one of the fundamental problems in computer science. For an integer $n>0$, the set $\{0,1,2, \cdots, n-1\}$ with the addition $(\bmod n)$ forms an Abelian group. Therefore, pursing efficient algorithms in the classical computing model for the basis of Abelian group has fundamental significance.

In this paper, we obtain an $O(n)$-time deterministic algorithm for finding the basis of an Abelian group with $n$ elements. This improves the previous $O\left(n^{2}\right)$ time and $O\left(n^{1.5}\right)$ time algorithms by Chen [3] and by Buchmann and Schmidt [2] respectively. We then derive an $O\left(\left(\sum_{i=1}^{k} p_{i}^{n_{i} / 2} n_{i}^{2}\right)(\log n) \log \log n\right)$-time randomized algorithm to compute the basis of Abelian group $G$ of size $n$ with factorization $n=p_{1}^{n_{1}} \cdots p_{t}^{n_{t}}$, which is also a part of the input. It implies an $O\left(n^{1 / 2}(\log n)^{3} \log \log n\right)$-time randomized algorithm to compute the basis of an Abelian group $G$ of size $n$. It also implies that if $n$ is an integer in $\{1,2, \cdots, m\}-G(m, c)$, then the basis of an Abelian group of size $n$ can be computed in $O\left((\log n)^{\frac{c}{2}+3} \log \log n\right)$-time, where $c$ is any positive constant and $G(m, c)$ is a subset of the small fraction of integers in set $\{1,2, \cdots, m\}$ with $\frac{|G(m, c)|}{m}=O\left(\frac{1}{(\log m)^{c / 2}}\right)$ for every integer $m$. Since saving the multiplication table of a group of size $n$ takes $O\left(n^{2}\right)$ space, the multiplication table of the Abelian group can be accessed as an oracle during the computation. In many applications, multiplication table is not necessary since the product of two elements can be calculated in running time.

\section{Notations}

For two positive integers $x$ and $y,(x, y)$ represents the greatest common divisor (GCD) between them. For a set $A,|A|$ denotes the number of elements in $A$. For a real number $x,\lfloor x\rfloor$ is the largest integer $\leq x$ and $\lceil x\rceil$ is the least integer $\geq x$. For two integers $x$ and $y, x \mid y$ means that $y=x c$ for some integer $c$.

A group is a nonempty set $G$ with a binary operation "." that is closed in set $G$ and satisfies the following properties (for simplicity, " $a b$ " represents " $a \cdot b$ "): 1) for every three elements $a, b$ and $c$ in $G, a(b c)=(a b) c$; 2)there exists an identity element $e \in G$ such that $a e=e a=a$ for every $a \in G$; 3)for every element $a \in G$, there exists $a^{-1} \in G$ with $a a^{-1}=a^{-1} a=e$. A group $G$ is finite if $G$ has only finite elements. Let $e$ be the identity element of $G$, i.e. $a e=a$ for each $a \in G$. For $a \in G$, ord $(a)$, the order of $a$, is the least integer $k$ such that $a^{k}=e$. For $a \in G$, define $\langle a\rangle$ to be the subgroup of $G$ generated by the element $a$ (in other words, $\langle a\rangle=\left\{e, a, a^{2}, \cdots, a^{\text {ord }(a)-1}\right\}$ ). Let $A$ and $B$ be two subsets of group $G$, define $A B=A \cdot B=A \circ B=\{a b \mid a \in A$ and $b \in B\}$.

A group $G$ is an Abelian group if $a b=b a$ for every pair of elements $a, b \in G$. Assume that $G$ is an Abelian group with elements $g_{1}, g_{2}, \cdots, g_{n}$. For each element $g_{i} \in G$, it corresponds to an index $i$. According to the theory of Abelian group, a finite Abelian group $G$ of $n$ elements can be represented as 
$G=G\left(p_{1}^{n_{1}}\right) \circ G\left(p_{2}^{n_{2}}\right) \circ \cdots \circ G\left(p_{t}^{n_{t}}\right)$, where $n=p_{1}^{n_{1}} p_{2}^{n_{2}} \cdots p_{t}^{n_{t}}, p_{1}<p_{2}<\cdots<p_{t}$ are the prime factors of $n$, and $G\left(p_{i}^{n_{i}}\right)$ is a subgroup of $G$ with $p_{i}^{n_{i}}$ elements (see [9]). We also use the notation $G_{p_{i}}$ to represent the subgroup of $G$ with size $p_{i}^{n_{i}}$. Any Abelian group $G$ of size $p^{m}$ can be represented by $G=G\left(p^{m_{1}}\right) \circ G\left(p^{m_{2}}\right) \circ \cdots \circ G\left(p^{m_{k}}\right)$, where $m=\sum_{i=1}^{k} m_{i}$ and $1 \leq m_{1} \leq m_{2} \leq \cdots \leq m_{k}$. Notice that each $G\left(p^{m_{i}}\right)$ is a cyclic group.

For, $a_{1}, a_{2}, \cdots, a_{k}$ from the Abelian group $G$, denote $\left\langle a_{1}, a_{2}, \cdots, a_{k}\right\rangle$ to be the set of all elements in $G$ generated by $a_{1}, \cdots, a_{k}$. In other words, $\left\langle a_{1}, a_{2}, \cdots, a_{k}\right\rangle=\left\langle a_{1}\right\rangle\left\langle a_{2}\right\rangle \cdots\left\langle a_{k}\right\rangle$. An element $a \in G$ is independent of $a_{1}, a_{2}, \cdots, a_{k}$ in $G$ if $a \neq e$ and $\left\langle a_{1}, a_{2}, \cdots, a_{k}\right\rangle \cap\langle a\rangle=\{e\}$. If $G=\left\langle a_{1}, a_{2}, \cdots, a_{k}\right\rangle$, then $\left\{a_{1}, a_{2}, \cdots, a_{k}\right\}$ is called a set of generators of $G$.

The elements $a_{1}, a_{2}, \cdots, a_{k}$ from the Abelian group $G$ are independent if $a_{i}$ is independent of $a_{1}, \cdots, a_{i-1}, a_{i+1}, \cdots, a_{k}$ for every $i$ with $1 \leq i \leq k$. A basis of $G$ is a set of independent elements $a_{1}, \cdots, a_{k}$ that can generate all elements of $G$ (in other words, $G=\left\langle a_{1}, a_{2}, \cdots, a_{k}\right\rangle$ ).

\section{3. $\quad$ Algorithm with $O(n \log n)$ Steps}

The algorithm in this section has two parts. The first part decomposes an Abelian group into product $G\left(p_{1}^{n_{1}}\right) \circ G\left(p_{2}^{n_{2}}\right) \circ \cdots \circ G\left(p_{k}^{n_{k}}\right)$. In order to get the subgroup of size $p_{i}^{n_{i}}$, we find the set of elements with the order of $p_{i}$-power.

The second part finds the basis of each group $G\left(p_{i}^{n_{i}}\right)$. The algorithm has several stages and each stage finds a member of basis at a time for $G\left(p_{i}^{n_{i}}\right)$. Assume that $b_{1}, \cdots, b_{h}$, which $\operatorname{satisfy} \operatorname{ord}\left(b_{1}\right) \geq$ $\operatorname{ord}\left(b_{2}\right) \geq \cdots \geq \operatorname{ord}\left(b_{h}\right)$, are the elements of a basis of the Abelian group $G\left(p^{u}\right)$. We will find another set of the basis $a_{1}, \cdots, a_{h}$. The element $a_{1}$ is selected among all elements in $G\left(p^{u}\right)$ such that $a_{1}$ has the largest order ord $\left(a_{1}\right)$. Therefore, ord $\left(a_{1}\right)=\operatorname{ord}\left(b_{1}\right)$. Assume that $a_{1}, \cdots, a_{k}$ have been obtained such that $\operatorname{ord}\left(a_{1}\right)=\operatorname{ord}\left(b_{1}\right), \cdots, \operatorname{ord}\left(a_{k}\right)=\operatorname{ord}\left(b_{k}\right)$. We show that it is always possible to find another $a_{k+1}$ such that $\left(\left\langle a_{1}\right\rangle \cdots\left\langle a_{k}\right\rangle\right) \cap\left\langle a_{k+1}\right\rangle=\{e\}$ and $\operatorname{ord}\left(a_{k+1}\right)=\operatorname{ord}\left(b_{k+1}\right)$. The possibility of such an extension is shown at Lemma 4 and Lemma 7. We maintain a subset $M$ of elements of $G\left(p^{u}\right)$ such that $M$ consists of all elements $a \in G$ that are independent of $a_{1}, a_{2}, \cdots, a_{k}$ and $\operatorname{ord}(a) \leq \operatorname{ord}\left(a_{k}\right)$. We search for $a_{k+1}$ from $M$ by selecting the element with the highest order. After $a_{k+1}$ is found, $M$ will be updated.

In this section, we develop an $O(n \log n)$ time algorithm to compute the basis of a finite Abelian group. The algorithm and its proof are self-contained. In section 4 , we improve this algorithm to be in linear time by using a result of Kavitha [12]. For an integer $n$, it can be factorized into product of primer numbers in $O\left(\sqrt{n}(\log n)^{2}\right)$ time by the brute force method. Both this section and section 4 spend at least linear time for computing the basis of an Abelian group. Therefore, we always assume that the primer factorization of $n$, which is the size of input Abelian group, is known in the two sections.

Lemma 1 ([24]). There exists an $O(n \log n)$ time algorithm such that given a group $G$ of size $n$, it computes the order of all elements $g$ with $\operatorname{ord}(g)=p_{i}^{j}$ for some $p_{i} \| G \mid$ and $j \geq 0$.

Proof: $\quad$ Assume that $n$ has the primer factorization $n=p_{1}^{n_{1}} p_{2}^{n_{2}} \cdots p_{t}^{n_{t}}$ and $n_{i} \geq 1$ for $i=1,2, \cdots, t$. Given the multiplication table of $G$, with $O(\log m)$ steps, we can compute $a^{m}$. This can be done by a straightforward divide and conquer method with the recursion $a^{m}=a^{\frac{m}{2}} \cdot a^{\frac{m}{2}}$ if $m$ is even or $a^{m}=a \cdot a^{\left\lfloor\frac{m}{2}\right\rfloor} \cdot a^{\left\lfloor\frac{m}{2}\right\rfloor}$ if $m$ is odd.

For each prime factor $p_{i}$ of $n$, compute $a^{p_{i}}$ for each $a \in G$. Build the table $T_{i}$ so that $T_{i}(a)=a^{p_{i}}$ for $a \in G$. The table $T_{i}$ can be built in $O\left(n \log p_{i}\right)$ steps.

For each $a \in G$ and prime factor $p_{i}$ of $n$, try to find the least integer $j$, which may not exist, such that $a^{p_{i}^{j}}=e$. It takes $O\left(n_{i}\right)$ steps by looking up the table $T_{i}$. For each $p_{i}$, trying all $a \in G \operatorname{takes} O\left(n\left(\log p_{i}+n_{i}\right)\right)$ steps. Therefore, the total time is $O\left(n\left(\sum_{i=1}^{t}\left(\log p_{i}+n_{i}\right)\right)=O(n \log n)\right.$.

Lemma 2. Assume $G$ is an Abelian group of size $n$. We have the following two facts: 1) If $n=m_{1} m_{2}$ with $\left(m_{1}, m_{2}\right)=1, G^{\prime}=\left\{a \in G \mid a^{m_{1}}=e\right\}$ and $G^{\prime \prime}=\left\{a^{m_{1}} \mid a \in G\right\}$, then both $G^{\prime}$ and $G$ are subgroups of $G$, $G=G^{\prime} \circ G^{\prime \prime},\left|G^{\prime}\right|=m_{1}$ and $\left|G^{\prime \prime}\right|=m_{2}$. Furthermore, for every $a \in G$, if $\left(\operatorname{ord}(a), m_{1}\right)=1$, then $a \in G^{\prime \prime}$. 2)If $n=p_{1}^{n_{1}} p_{2}^{n_{2}} \cdots p_{t}^{n_{t}}$, then $G=G\left(p_{1}^{n_{1}}\right) \circ G\left(p_{2}^{n_{2}}\right) \circ \cdots \circ G\left(p_{t}^{n_{t}}\right)$, where $G\left(p_{i}^{n_{i}}\right)=\left\{a \in G \mid a^{p_{i}^{n_{i}}}=e\right\}$ for $i=1, \cdots, t$. 
Proof: It is easy to verify that $G^{\prime}$ is subgroup of $G$. Assume $a_{1}, \cdots, a_{s_{1}}, b_{1}, \cdots, b_{s_{2}}$ are the elements in a basis of $G$ such that $\operatorname{ord}\left(a_{i}\right) \mid m_{1}$ for $i=1, \cdots, s_{1}$ and $\operatorname{ord}\left(b_{j}\right) \mid m_{2}$ for $j=1, \cdots, s_{2}$. It is easy to see that $a_{i}^{m_{1}}=e$ for $i=1, \cdots, s_{1}$ and $b_{j}^{m_{1}} \neq e$ for $j=1, \cdots, s_{2}$. For each $b_{j},\left\langle b_{j}\right\rangle=\left\langle b_{j}^{m_{1}}\right\rangle$ since $\left(m_{1}, m_{2}\right)=1$ and $\operatorname{ord}\left(b_{j}\right) \mid m_{2}$. Assume that $x=a^{m_{1}}$ and $y=a^{\prime m_{1}}$. Both $x$ and $y$ belong to $G^{\prime \prime}$. Let's consider $x y=\left(a a^{\prime}\right)^{m_{1}}$. We still have $x y \in G^{\prime \prime}$. Thus, $G^{\prime \prime}$ is closed under multiplication. Since $G^{\prime \prime}$ is a subset of a finite group, $G^{\prime \prime}$ is a group. Therefore, $G^{\prime \prime}$ is a group generated by $b_{1}^{m_{1}}, \cdots, b_{s_{2}}^{m_{1}}$ that is the same as the group generated by $b_{1}, \cdots, b_{s_{2}}$. Therefore, $G^{\prime \prime}$ is of size $m_{2}$. On the other hand, $G^{\prime}$ has basis of elements $a_{1}, \cdots, a_{s_{1}}$ and is of size $m_{1}$. We also have that $G^{\prime} \cap G^{\prime \prime}=\{e\}$. It is easy to see that $G=G^{\prime} \circ G^{\prime \prime}$. For $a \in G$ with $\left(\operatorname{ord}(a), m_{1}\right)=1$, $\left\langle a^{m_{1}}\right\rangle=\langle a\rangle$ and $a^{m_{1}} \neq e$. So, we have $a^{m_{1}} \in G^{\prime \prime}$, which implies that $a \in\langle a\rangle=\left\langle a^{m_{1}}\right\rangle \subseteq G^{\prime \prime}$. Part 2) follows from part 1).

Lemma 3. Assume $G$ is a group of size $n=p_{1}^{n_{1}} p_{2}^{n_{2}} \cdots p_{t}^{n_{t}}$. Given the table of the orders of all elements $g \in G$ with $\operatorname{ord}(g)=p_{i}^{j}$ for some $p_{i}$ and $j \geq 0$, with $O(n)$ steps, $G$ can be decomposed as the product of subgroups $G\left(p_{1}^{n_{1}}\right) \circ \cdots \circ G\left(p_{t}^{n_{t}}\right)$.

Proof: By Lemma 2, the elements of each $G\left(p_{i}^{n_{i}}\right)$ consists of all elements of $G$ with order $p_{i}^{j}$ for some integer $j \geq 0$. Therefore, we have the following algorithm:

Compute the list of integers $p_{1}, p_{1}^{2}, \cdots, p_{1}^{n_{1}}, p_{2}, p_{2}^{2}, \cdots, p_{2}^{n_{2}}, \cdots, p_{t}, p_{t}^{2}, \cdots, p_{t}^{n_{t}}$. This can be done in $O(\log n)^{2}$ steps because $n_{1}+n_{2}+\cdots+n_{t} \leq \log n$. Also sort those integers $p_{1}, p_{1}^{2}, \cdots, p_{1}^{n_{1}}, p_{2}, p_{2}^{2}, \cdots, p_{2}^{n_{2}}, \cdots$, $p_{t}, p_{t}^{2}, \cdots, p_{t}^{n_{t}}$ by increasing order. It takes $(\log n)^{2}$ steps because bubble sorting those $\log n$ integers takes $O\left((\log n)^{2}\right)$ steps. Let $q_{1}<q_{2} \cdots<q_{m}$ be the list of integers sorted from $p_{1}, p_{1}^{2}, \cdots, p_{1}^{n_{1}}, p_{2}, p_{2}^{2}, \cdots, p_{2}^{n_{2}}, \cdots$, $p_{t}, p_{t}^{2}, \cdots, p_{t}^{n_{t}}$.

Set up the array $A$ of $n$ buckets. Put all elements of order $k$ into bucket $A[k]$. Merge the buckets $A\left[p_{i}\right], A\left[p_{i}^{2}\right], \cdots, A\left[p_{i}^{n_{i}}\right]$ to obtain $G\left(p_{i}^{n_{i}}\right)$. This can be done by scanning the array $A$ from left to right once and fetching the elements from the array $A\left[\right.$ ] at those positions $q_{1}<q_{2} \cdots<q_{m}$.

The following lemma is essential from Chen's early work [3]. Its proof, which was written in Chinese, is refined here.

Lemma 4 ([3]). Let $G$ be an Abelian group of size $p^{t}$ for prime $p$ and integer $t \geq 1$. Assume $a_{1}, a_{2}, \cdots, a_{k}$ are independent elements in $G$ and $b$ is also an elements in $G$ with $\operatorname{ord}(b) \leq \operatorname{ord}\left(a_{i}\right)$ for $i=1, \cdots, k$. Then there exists $b^{\prime} \in\left\langle a_{1}, \cdots, a_{k}, b\right\rangle$ with $\operatorname{ord}\left(b^{\prime}\right) \mid \operatorname{ord}(b)$ such that (1) $a_{1}, \cdots, a_{k}, b^{\prime}$ are independent elements in $G$; (2) $\left\langle a_{1}, \cdots, a_{k}, b^{\prime}\right\rangle=\left\langle a_{1}, \cdots, a_{k}, b\right\rangle$; and (3) $b^{\prime}$ can be expressed as $b^{\prime}=b \prod_{i=1}^{k}\left(a_{i}^{-t_{i} p^{\xi_{i}-\eta}}\right)$, where $\eta$ is the least integer that $b^{p^{\eta}} \in\left\langle a_{1}, \cdots, a_{k}\right\rangle$.

Proof: $\quad$ Let $\operatorname{ord}\left(a_{i}\right)=p^{n_{i}}$ and $\operatorname{ord}(b)=p^{m}, n_{i} \geq m$ for $i=1, \ldots, k$. Let $\left\langle a_{1}, \cdots, a_{k}\right\rangle \cap\langle b\rangle=\langle c\rangle$. We assume that $c \neq e$ (Otherwise, let $b^{\prime}=b$ and finish the proof). Assume,

$$
c=a_{1}^{t_{1} p^{\xi_{1}}} \cdots a_{k}^{t_{k} p^{\xi_{k}}}=b^{h p^{\eta}}
$$

where $0 \leq t_{i}<p^{n_{i}-\xi_{i}}$ and $\left(t_{i}=0\right.$ or $\left.\left(t_{i}, p\right)=1\right)$ for $i=1, \cdots, k$ and $0<h<p^{m-\eta}$ with $(h, p)=1$ and $\eta<m$ (because $c \neq e$ ).

Since $\left(t_{i}, p\right)=1$, the order of each $a_{i}^{t_{i} p^{\xi_{i}}}$ is $\frac{p^{n_{i}}}{p^{\xi_{i}}}$. The order of $a_{1}^{t_{1} p^{\xi_{1}}} \cdots a_{k}^{t_{k} p^{\xi_{k}}}$ is $\max \left\{\frac{p^{n_{i}}}{p^{\xi_{i}}} \mid t_{i} \neq 0\right.$, and $i=$ $1, \ldots, k\}$. On the hand, the order of $b^{h p^{\eta}}$ is $\frac{p^{m}}{p^{\eta}}$. Thus, we have $\max \left\{\frac{p^{n_{i}}}{p^{\xi_{i}}} \mid t_{i} \neq 0\right.$, and $\left.i=1, \ldots, k\right\}=\frac{p^{m}}{p^{\eta}}$. Therefore, $p^{n_{i}-\xi_{i}} \leq p^{m-\eta}$ for each $i=1, \cdots, k$. Thus, we have $n_{i}-\xi_{i} \leq m-\eta$. Since $(h, p)=1$, we have $\left\langle b^{h p^{\eta}}\right\rangle=\left\langle b^{p^{\eta}}\right\rangle$. Without loss of generality, we assume that $h=1$. It is easy to see that $\eta$ is the least integer such that $b^{p^{\eta}} \in\left\langle a_{1}, \cdots, a_{k}\right\rangle$. We have $\xi_{i} \geq \eta+\left(n_{i}-m\right) \geq \eta$ for $i=1, \ldots, k$. Let

$$
b^{\prime}=\prod_{i=1}^{k}\left(a_{i}^{-t_{i} p^{\xi_{i}-\eta}}\right) \cdot b .
$$

Clearly, $b^{\prime} \in \prod_{i=1}^{k}\left\langle a_{i}\right\rangle \cdot\langle b\rangle$. By (1) and the fact $h=1, b^{p^{\eta}}=\left(\prod_{i=1}^{k} a_{i}^{t_{i} p^{\xi_{i}-\eta}}\right)^{p^{\eta}}$. By (2), we have $b^{\prime p^{\eta}}=e$, which implies ord $\left(b^{\prime}\right) \mid p^{\eta}$. We obtain the following:

$$
\left\langle a_{1}, \cdots, a_{k}, b\right\rangle=\left\langle a_{1}, \cdots, a_{k}, b^{\prime}\right\rangle .
$$


We now want to prove that $\left\langle a_{1}, \cdots, a_{k}\right\rangle \cap\left\langle b^{\prime}\right\rangle=\{e\}$.

If, on the contrary, $\left\langle a_{1}, \cdots, a_{k}\right\rangle \cap\left\langle b^{\prime}\right\rangle=\left\langle c^{\prime}\right\rangle$ and $c^{\prime} \neq e$. We assume $c^{\prime}=b^{p^{u \eta^{\prime}}}$ for some $u$ with $(u, p)=1$. Since $\left\langle b^{p^{u \eta^{\prime}}}\right\rangle=\left\langle b^{p^{\eta^{\prime}}}\right\rangle$, let $u=1$. There exist integers $s_{i}, \xi_{i}^{\prime}(i=1, \cdots, k)$ such that

$$
c^{\prime}=\prod_{i=1}^{k} a_{i}^{s_{i} p^{\xi_{i}^{\prime}}}=b^{\prime p^{\eta^{\prime}}}=\prod_{i=1}^{k} a_{i}^{-t_{i} p^{\xi_{i}-\eta+\eta^{\prime}}} \cdot b^{p^{\eta^{\prime}}},
$$

where $0 \leq \xi_{i}^{\prime}<n, 0 \leq \eta^{\prime}<\eta$. If $\eta^{\prime} \geq \eta$, we have $c^{\prime}=e$ by (1), (2), and (3). This contradicts the assumption $c^{\prime} \neq e$.

Since $c=b^{p^{\eta}} \neq e$, we have $b^{p^{\eta^{\prime}}} \neq e$. Since $\left\langle a_{1}, \cdots, a_{k}\right\rangle \cap\langle b\rangle=\left\langle b^{p^{\eta}}\right\rangle$ and $\eta>\eta^{\prime}$, we have $b^{p^{\eta^{\prime}}} \notin$ $\left\langle a_{1}, \cdots, a_{k}\right\rangle \cap\langle b\rangle$. By $(3)$,

$$
b^{p^{\eta^{\prime}}}=\prod_{i=1}^{k} a_{i}^{s_{i} p^{\xi_{i}^{\prime}}} \cdot \prod_{i=1}^{k} a_{i}^{t_{i} p^{\xi_{i}-\eta+\eta^{\prime}}}
$$

By (4), we also have $b^{p^{\eta^{\prime}}} \in\left\langle a_{1}, \cdots, a_{k}\right\rangle \cap\langle b\rangle$. This contradicts that $\eta$ is the least integer such that $b^{p^{\eta}} \in$ $\left\langle a_{1}, \cdots, a_{k}\right\rangle$ (notice that $\eta^{\prime}<\eta$ ). Thus, $\left\langle a_{1}, \cdots, a_{k}\right\rangle \cap\left\langle b^{\prime}\right\rangle=\{e\}$.

Definition 5. Assume that group $G$ has basis $b_{1}, \cdots, b_{t}$ with $\operatorname{ord}\left(b_{1}\right) \geq \cdots \geq \operatorname{ord}\left(b_{t}\right)$.

- Assume that $a_{1}, \cdots, a_{k}$ and $b$ are the same as those in Lemma 4 . We use independent-extension $\left(a_{1}, \cdots, a_{k}, b\right)$ to represent $b^{\prime}$ derived in the Lemma 4 such that (1) $a_{1}, \cdots, a_{k}, b^{\prime}$ are independent elements in $G$; and (2) $\left\langle a_{1}, \cdots, a_{k}, b^{\prime}\right\rangle=\left\langle a_{1}, \cdots, a_{k}, b\right\rangle$.

- Let $a_{1}, \cdots, a_{k}$ be the elements of $G$ with $\operatorname{ord}\left(a_{1}\right)=\operatorname{ord}\left(b_{1}\right), \cdots, \operatorname{ord}\left(a_{k}\right)=\operatorname{ord}\left(b_{k}\right)$ and $\left(\prod_{i \neq j}\left\langle a_{i}\right\rangle\right) \cap$ $\left\langle a_{j}\right\rangle=\{e\}$ for every $j=1, \cdots, k$. Then $a_{1}, \cdots, a_{k}$ is called a partial basis of $G$. If $C\left(a_{1}, \cdots, a_{k}\right)=\{a \in$ $G \mid\left\langle a_{1}, \cdots, a_{k}\right\rangle \cap\langle a\rangle=\{e\}$ and $\left.\operatorname{ord}(a) \leq \operatorname{ord}\left(a_{k}\right)\right\}$, then $C\left(a_{1}, \cdots, a_{k}\right)$ is called a complementary space of the partial basis $a_{1}, \cdots, a_{k}$.

It is well known that the decomposition of Abelian group is unique (see [9]). For the completeness purpose, we prove the following lemma.

Lemma 6. Let $G$ be an Abelian group of size $p^{m}$ for some prime $p$ and integer $m$. Let $b_{1}, \cdots, b_{t}$ be a basis of $G$ with $\operatorname{ord}\left(b_{1}\right) \geq \cdots \geq \operatorname{ord}\left(b_{t}\right)$ and $b_{1}^{\prime}, \cdots, b_{t^{\prime}}^{\prime}$ be another basis of $G$ with $\operatorname{ord}\left(b_{1}^{\prime}\right) \geq \cdots \geq \operatorname{ord}\left(b_{t^{\prime}}^{\prime}\right)$. Then $t=t^{\prime}$ and $\operatorname{ord}\left(b_{1}\right)=\operatorname{ord}\left(b_{1}^{\prime}\right), \cdots, \operatorname{ord}\left(b_{t}\right)=\operatorname{ord}\left(b_{t}^{\prime}\right)$.

Proof: Assume that $i$ be the least integer that $\operatorname{ord}\left(b_{i}\right) \neq \operatorname{ord}\left(b_{i}^{\prime}\right)$. Without loss of generality, we assume that $\operatorname{ord}\left(b_{i}\right)>\operatorname{ord}\left(b_{i}^{\prime}\right)$. Let $h=\operatorname{ord}\left(b_{i}^{\prime}\right)$. Consider the generators set $\left\{b_{1}^{h}, b_{2}^{h}, \cdots, b_{t}^{h}\right\}$, which generates a subgroup of $G$ with $\prod_{j=1}^{i} p^{\operatorname{ord}\left(b_{j}\right)-h}$ elements. On the other hand, generator set $\left\{b_{1}^{\prime h}, b_{2}^{\prime h}, \cdots, b_{t^{\prime}}^{\prime h}\right\}$, which generates a subgroup of $G$ with $\prod_{j=1}^{i} p^{\text {ord }\left(b_{j}^{\prime}\right)-h}=\prod_{j=1}^{i-1} p^{\text {ord }\left(b_{j}^{\prime}\right)-h}=\prod_{j=1}^{i-1} p^{\text {ord }\left(b_{j}\right)-h}$ elements. Both sets generate the subgroup $\left\{a^{h}: a \in G\right\}$. This is a contradiction.

Lemma 7. Let $a_{1}, \cdots, a_{k}$ be partial basis of the Abelian $G$ with $p^{i}$ elements for some prime $p$ and integer $i \geq 0$. Then 1) $G$ can be generated by $\left\{a_{1}, \cdots, a_{k}\right\} \cup C\left(a_{1}, \cdots, a_{k}\right)$; and 2)the partial basis $a_{1}, \cdots, a_{k}$ can be extended to another partial basis $a_{1}, \cdots, a_{k}, a_{k+1}$ with complementary space $C\left(a_{1}, \cdots, a_{k}, a_{k+1}\right)=$ $\left\{a \in C\left(a_{1}, \cdots, a_{k}\right) \mid\left\langle a_{1}, \cdots, a_{k}, a_{k+1}\right\rangle \cap\langle a\rangle=\{e\}\right.$ and $\left.\operatorname{ord}(a) \leq \operatorname{ord}\left(a_{k+1}\right)\right\}$, and $a_{k+1}$ is the element of $C\left(a_{1}, \cdots, a_{k}\right)$ having the largest order ord $\left(a_{k+1}\right)$.

Proof: Assume group $G$ has the basis $b_{1}, \cdots, b_{t}$ with $\operatorname{ord}\left(b_{1}\right) \geq \cdots \geq \operatorname{ord}\left(b_{t}\right)$. 1$)$ We prove it by using induction. It is trivial at the case $k=0$. Assume that it is true at $k$. We consider the case at $k+1$. Let $a_{1}, \cdots, a_{k}, a_{k+1}$ be the elements of a partial basis of $G$. Let the $C\left(a_{1}, \cdots, a_{k}\right)$ be the complementary space for $a_{1}, \cdots, a_{k}$. By assumption, $G$ can be generated by $\left\{a_{1}, \cdots, a_{k}\right\} \cup C\left(a_{1}, \cdots, a_{k}\right)$. 
By the definition of partial basis (see Section 2), it is easy to see that $a_{k+1} \in C\left(a_{1}, \cdots, a_{k}\right)$. Select $a_{k+1}^{\prime}$ from $C\left(a_{1}, \cdots, a_{k}\right)$ such that $\operatorname{ord}\left(a_{k+1}^{\prime}\right)=\max \left\{\operatorname{ord}(a): a \in C\left(a_{1}, \cdots, a_{k}\right)\right\}$. By Lemma 4, independent-extension $\left(a_{1}, \cdots, a_{k}, a_{k+1}^{\prime}, b\right) \in C\left(a_{1}, \cdots, a_{k}, a_{k+1}^{\prime}\right)$ for each $b \in C\left(a_{1}, \cdots, a_{k}\right)$. We still have such a property that $\left\{a_{1}, \cdots, a_{k}, a_{k+1}^{\prime}\right\} \cup C\left(a_{1}, \cdots, a_{k}, a_{k+1}^{\prime}\right)$ can generate $G$. Thus, $a_{1}, \cdots, a_{k}$ can be extended into the basis of $G$ : $a_{1}, \cdots, a_{k}, a_{k+1}^{\prime}, \cdots, a_{t^{\prime}}^{\prime}$ with ord $\left(a_{1}\right) \geq \operatorname{ord}\left(a_{2}\right) \geq \cdots \geq \operatorname{ord}\left(a_{k}\right) \geq \operatorname{ord}\left(a_{k+1}^{\prime}\right) \geq$ $\cdots \geq \operatorname{ord}\left(a_{t^{\prime}}\right)$ by repeating the method above. Since the decomposition of $G$ has a unique structure (see Lemma 6), we have that $t=t^{\prime}, \operatorname{ord}\left(a_{1}\right)=\operatorname{ord}\left(b_{1}\right), \cdots, \operatorname{ord}\left(a_{k}\right)=\operatorname{ord}\left(b_{k}\right), \operatorname{ord}\left(a_{k+1}^{\prime}\right)=\operatorname{ord}\left(b_{k+1}\right), \cdots$, and $\operatorname{ord}\left(a_{t}^{\prime}\right)=\operatorname{ord}\left(b_{t}\right)$. Therefore, $\operatorname{ord}\left(a_{k+1}^{\prime}\right)=\operatorname{ord}\left(b_{k+1}\right)=\operatorname{ord}\left(a_{k+1}\right)$. Thus, we can select $a_{k+1}$ instead of $a_{k+1}^{\prime}$ to extend the partial basis from $a_{1}, \cdots, a_{k}$ to $a_{1}, \cdots, a_{k}, a_{k+1}$.

2) Notice that $C\left(a_{1}, \cdots, a_{k}, a_{k+1}\right) \subseteq C\left(a_{1}, \cdots, a_{k}\right)$. It follows from the proof of 1$)$.

Lemma 8. With $O(m)$ steps, one can compute $a^{p}$ for all elements a of group $G$, where $|G|=m=p^{i}$ elements for some prime $p$ and integer $i \geq 0$.

Proof: Initially mark all elements of $G-\{e\}$ "unprocessed" and mark the unit element $e$ "processed". We always select an unprocessed element $a \in G$ and compute $a^{p}$ until all elements in $G$ are processed. Compute $a^{p}$, which takes $O(\log p)$ steps, and its order ord $(a)=p^{j}$ by $\operatorname{trying} a^{p}, a^{p^{2}}, \cdots, a^{p^{j}}$, which takes $O\left(j^{2} \log p\right)=O\left(\left(\log p^{j}\right)^{2}\right)$ steps. Process $a^{k}$ according to the order $k=1,2, \cdots, p^{j}$, compute $\left(a^{k}\right)^{p}=\left(a^{p}\right)^{k}$ in $O\left(p^{j}\right)$ steps and mark $a, a^{2}, \cdots, a^{p^{j}}$ "processed". For each $k$ with $1 \leq k \leq p^{j}$ and $(k, p)=1$, $a^{k}$ is not processed before because the subgroups generated by $a^{k}$ and $a$ are the same (In other words, $\left\langle a^{k}\right\rangle=\langle a\rangle$ ). There are $p^{j}-p^{j-1} \geq \frac{p^{j}}{2}$ integers $k$ in the interval $\left[1, p^{j}\right]$ to have $(k, p)=1$. Therefore, we process at least $\frac{p^{j}}{2}$ new elements $a^{k}$ in $O\left(p^{j}\right)$ steps by computing $a^{k p}$ from $a^{p}$. Therefore, the total number of steps is $O(m)$.

Lemma 9. With $O(m)$ steps, one can compute $a^{\frac{\operatorname{ord}(a)}{p}}$ and $\log _{p} \operatorname{ord}(a)$ for all elements a of group $G$ with $|G|=m=p^{i}$ for some prime $p$ and integer $i \geq 0$.

Proof: We first prove that for any two elements $a, b \in G$, if $a^{p^{j}}=b$ for some $j \geq 0$ and ord $(b)=p^{t}$ for some $t \geq 1$, then $\operatorname{ord}(a)=p^{j+t}$. Assume that $\operatorname{ord}(a)=p^{s}$. First we should notice the number $j$ for $a^{p^{j}}=b$ is unique. Otherwise, $a^{p^{k}} \neq e$ for any integer $k$. This contradicts ord $(a) \mid p^{i}$. Assume $a^{p^{j_{1}}}=a^{p^{j_{2}}}=b \neq e$ for some $j_{1}<j_{2}$. Then we have $\left(a^{p^{j_{1}}}\right)^{p^{j_{2}-j_{1}}}=a^{p^{j_{1}}} \neq e$. The loop makes $a^{p^{k}} \neq e$ for every $k \geq 0$.

We have $a^{p^{j+t}}=\left(a^{p^{j}}\right)^{p^{t}}=b^{p^{t}}=e$. Therefore, $s \leq j+t$. Since $a^{p^{j}}=b \neq e$ and $\operatorname{ord}(a)=p^{s}$, we have $j<s$. $b^{p^{s-j}}=\left(a^{p^{j}}\right)^{s-j}=a^{p^{s}}=e$. Since $\operatorname{ord}(b)=p^{t}, t \leq s-j$ and $t+j \leq s$. Thus, we have $s=t+j$. Therefore, ord $(a)=p^{j+t}$. This implies that if $a^{p^{j}}=b \neq e$ for some $j$, then $a^{\frac{\operatorname{ord}(a)}{p}}=b^{\frac{\operatorname{ord}(b)}{p}}$ and $\log _{p}(\operatorname{ord}(a))=\log _{p}(\operatorname{ord}(b))+j$. This fact is used in the algorithm design.

By Lemma 8, we can have a table $P$ with $P(a)=a^{p}$ in $O(m)$ time. Assign flag -1 to each element in the group $G$ in the first step. If an element $a$ has its values $a^{\frac{\operatorname{ord}(a)}{p}}$ and $\log _{p} \operatorname{ord}(a)$ computed, its flag is changed to +1 . We maintain the table that always has the property that if $a^{\frac{\operatorname{ord}(a)}{p}}$ and $\log _{p} \operatorname{ord}(a)$ are available (the flag of $a$ is +1$)$, then $b^{\frac{\operatorname{ord}(b)}{p}}$ and $\log _{p} \operatorname{ord}(b)$ are available for every $b=a^{p^{j}}$ for some $j>0$. For an element $b$ of order $p^{t}$, when computing $b^{\frac{\operatorname{ord}(b)}{p}}=b^{p^{t-1}}$, we also compute $b_{i}^{\frac{\operatorname{ord}\left(b_{i}\right)}{p}}$ and $\log _{p} \operatorname{ord}\left(b_{i}\right)$ for $b_{i}=b^{p^{i}}$ with $i=1,2, \cdots, t-1$ until it meets some $b_{i}$ with flag +1 . The element $b_{i}=b_{i-1}^{p}$ can be computed in $O(1)$ steps from $b_{i-1}$ since table $P$ is available. It is easy to see that such a property of the table is always maintained. Thus, the time is proportional to the number of elements with flag +1 . The total time is $O(m)$.

Assume the Abelian group $G$ has $p^{j}$ elements. By Lemma 9, we can set up an array $U[$ ] of $m$ buckets that each its position $U\left[g_{i}\right]$ contains all the elements $a$ of $G$ with $a^{\frac{\operatorname{ord}(a)}{p}}=g_{i}$. We also maintain a double linked list $M$ that contains all of the elements of $G$ with order from small to large in the first step.

Definition 10. Assume $a_{1}, a_{2}, \cdots, a_{k}, a_{k+1}$ are elements of Abelian group $G$ with $p^{t}$ elements for some prime $p$ and integer $t \geq 0$. 
- Define $L\left(a_{1}, \cdots, a_{k}\right)=\left\langle a_{1}^{\frac{\operatorname{ord}\left(a_{1}\right)}{p}}, \cdots, a_{k}^{\frac{\operatorname{ord}\left(a_{k}\right)}{p}}\right\rangle-\{e\}$.

- If $A=\left\{a_{1}, \cdots, a_{k}\right\}$, define $L(A)=L\left(a_{1}, \cdots, a_{k}\right)$.

Lemma 11. Assume $a_{1}, a_{2}, \cdots, a_{k}, a_{k+1}$ are independent elements of $G$, which has $p^{t}$ elements for some prime $p$ and integer $t \geq 0$. Then 1) $L\left(a_{1}, \cdots, a_{k}, a_{k+1}\right)=L\left(a_{1}, \cdots, a_{k}\right) \cup\left(L\left(a_{k+1}\right) \cup\left(L\left(a_{k+1}\right) \circ L\left(a_{1}, \cdots, a_{k}\right)\right)\right)$, and 2) $L\left(a_{1}, \cdots, a_{k}\right) \cap\left(L\left(a_{k+1}\right) \cup\left(L\left(a_{k+1}\right) \circ L\left(a_{1}, \cdots, a_{k}\right)\right)\right)=\emptyset$.

Proof: To prove 1) in the lemma, we just need to follow the definition of $L($ ). For 2), we use the condition $\left\langle a_{k+1}\right\rangle \cap\left\langle a_{1}, a_{2}, \cdots, a_{k}\right\rangle=\{e\}$ since $a_{1}, a_{2}, \cdots, a_{k}$ are independent (see the definition at Section 2).

The procedure of obtaining $L$ is shown in the following algorithm, which is also used to find the basis of the Abelian group of size power of a prime in Lemma 12.

\section{Algorithm A}

Input:

an Abelian group $G$ with size $p^{t}$, prime $p$ and integer $t$,

a table $T$ with $T(a)=a^{\frac{\operatorname{ord}(a)}{p}}$ for each $a \neq e$,

a table $R$ with $R(a)=j$ if $\operatorname{ord}(a)=p^{j}$ for each $a \in G$,

an array of buckets $U$ with $U(b)=\{a \mid T(a)=b\}$.

a double linked list $M$ that contains all elements $a$ of $G$ with nondecreasing order by $\operatorname{ord}(a)$

(each element $a \in G$ has a pointer to the node $N$, which holds $a$, in $M$ ).

Output: the basis of $G$;

begin

$L=\emptyset ; B=\emptyset ;$

repeat

select $a \in M$ with the largest ord $(a)$ ( $a$ is at the end of the double linked list $M$ );

$B=B \cup\{a\}$;

$L^{\prime}=L(a) \cup(L(a) \circ L)$

for (each $b \in L^{\prime}$ ) remove all elements in $U(b)$ from $M$;

$L=L \cup L^{\prime}$

until $\left(\sum_{a_{j} \in B} R\left(a_{j}\right)=t\right)$;

output the set $B$ as the basis of $G$;

end

End of Algorithm A

Lemma 12. There is an $O(m)$ time algorithm for computing the basis of an $G$ group with $m=p^{t}$ elements for some prime $p$ and integer $t \geq 0$.

Proof: Algorithm A is described above the lemma. By Lemma 8, we can obtain the orders of all elements of $G$ in $O(m)$ time. With another $O(m)$ time for Bucket sorting (see [5]), we can set up the double linked list $M$ that contains all elements $a$ of $G$ with nondecreasing order by ord $(a)$. By Lemma 9, with $O(m)$ steps, we can obtain the table $T$ and table $R$ with $T(a)=a^{\frac{\operatorname{ord}(a)}{p}}$ and $R(a)=\log _{p} \operatorname{ord}(a)$ for each $a \neq e$ in $G$. With table $R$, we can obtain the array of buckets $U$ with $U(b)=\{a \mid T(a)=b\}$ for each $b \in G$ in $O(m)$ steps by Bucket sorting. The tables $T$ and $R$, bucket array $U$, and double linked list are used as the inputs of the algorithm.

For every element $b \in G$ with $b \neq e, \operatorname{ord}(b) \leq \min \left\{\operatorname{ord}\left(a_{i}\right) \mid i=1, \cdots, k\right\}$, and $\left\langle a_{1}, \cdots, a_{k}\right\rangle \cap\langle b\rangle \neq\{e\}$ iff $b^{\frac{\operatorname{ord}(b)}{p}}$ is in $L\left(a_{1}, \cdots, a_{k}\right)$. When a new $a_{k+1}$ is found, $L\left(a_{1}, a_{2}, \cdots, a_{k}\right)$ becomes to $L\left(a_{1}, a_{2}, \cdots, a_{k}, a_{k+1}\right)=$ $L\left(a_{1}, a_{2}, \cdots, a_{k}\right) \cup\left(L\left(a_{k+1}\right) \cup L\left(a_{k+1}\right) \circ L\left(a_{1}, a_{2}, \cdots, a_{k}\right)\right)$. For each new element $g_{i} \in L\left(a_{k+1}\right) \cup L\left(a_{k+1}\right) \circ$ $L\left(a_{1}, a_{2}, \cdots, a_{k}\right)=L\left(a_{1}, a_{2}, \cdots, a_{k}, a_{k+1}\right)-L\left(a_{1}, a_{2}, \cdots, a_{k}\right)$ (see Lemma 11), we obtain the bucket $U\left[g_{i}\right]$ that contains all elements $a \in G$ with $a^{\frac{\operatorname{ord}(a)}{p}}=g_{i}$. Then remove all elements of $U\left[g_{i}\right]$ from the double linked list $M$. This makes $M$ hold all elements of $C\left(a_{1}, \cdots, a_{k}, a_{k+1}\right)$ (see Definition 5). Removing an element takes $O(1)$ time and each element is removed at most once. Therefore, the total time is $O(m)$. It is easy to check the correctness of the algorithm by using Lemma 7 . 


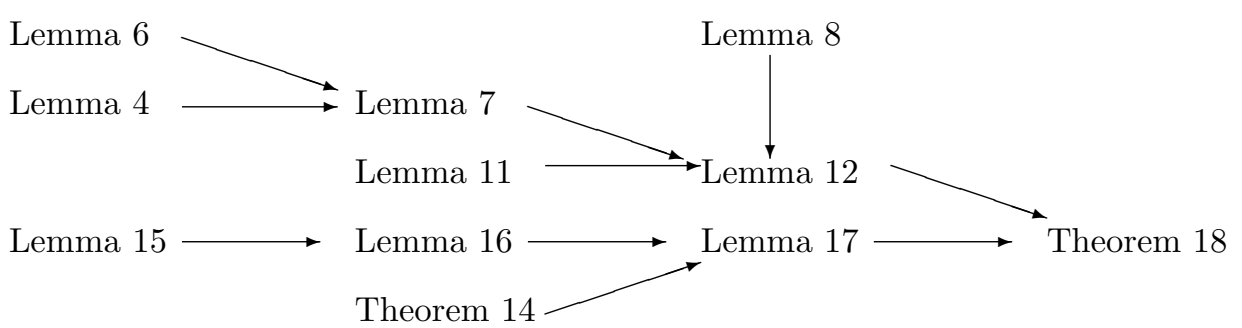

Figure 1: Structure for Proving Theorem 18

Theorem 13. There is an $O(n \log n)$ time algorithm for computing the basis of an Abelian $G$ group with $n$ elements.

Proof: Assume $n=p_{1}^{n_{1}} \cdot p_{2}^{n_{2}} \cdots p_{t}^{n_{t}}$. By Lemma 1 and Lemma 3, the group $G$ can be decomposed into product $G=G\left(p_{1}^{n_{2}}\right) \circ G\left(p_{2}^{n_{2}}\right) \circ \cdots \circ G\left(p_{t}^{n_{t}}\right)$ in $O(n \log n)$ steps. By Lemma 12, the basis of each $G\left(p_{i}^{n_{i}}\right)$ $(i=1,2, \cdots, t)$ can be found in $O\left(p_{i}^{n_{i}}\right)$ time. Thus, the total time is $O(n \log n)+O\left(\sum_{i=1}^{t} p^{n_{i}}\right)=O(n \log n)$.

\section{Algorithm in $O(n)$ Time}

In this section, we improve the running time from $O(n \log n)$ to $O(n)$ by using a result of Kavitha [12]. Kavitha's theorem is stated below:

Theorem 14 ([12]). Given any group $G$ of $n$ elements, one can compute the orders of all elements in $G$ in $O(n \log p)$ time, where $p$ is the smallest prime non-divisor of $n$.

In this section, we obtain a linear time group decomposition $G=G\left(p_{1}^{n_{1}}\right) \circ \cdots \circ G\left(p_{t}^{n_{t}}\right)$, where the Abelian group $G$ has $n$ elements with $n=p_{1}^{n_{1}} \cdots p_{t}^{n_{t}}$. The technique we use here is the following: For an Abelian group $G$ with $|G|=2^{n_{1}} m_{2}$, where $m_{2}$ is an odd number. We derive a decomposition of $G=G_{1} \circ G_{2}$ in linear time such that $\left|G_{1}\right|=2^{n_{1}}$ and $\left|G_{2}\right|=m_{2}$. Then we apply Kavitha's theorem to decompose the group $G_{2}$. In order to derive the elements of $G_{2}$, we convert this problem into a search problem in a special directed graph where each of its nodes has one outgoing edge. The directed graph has all elements of $G$ as its vertices. Vertex $a$ has edge going to vertex $b$ if $a^{2}=b$. Each weakly connected component of such a directed graph has a unique directed cycle. We show that each node in the cycle can be added to $G_{2}$. Removing the cycle nodes, we obtain a set of directed trees. The nodes that have a path of length at least $n_{1}$ to a leaf node can be also added to the group $G_{2}$. Searching the directed graph takes $O(n)$ time. Combining with Kavitha's theorem, we obtain the $O(n)$ time decomposition for the graph $G$. Using the result of section 3 , we obtain the $O(n)$ time algorithm for finding the basis. An $O(n)$ time algorithm for computing the orders of all elements in an Abelian group $G$ was recently reported by Kavitha [11]. The proof is more involved. Our linear time decomposition method using Theorem 14 is also technically interesting as it converts an algebraic problem into a searching problem in a directed graph that every node has exactly one outgoing edge. Using Theorem 14, our method is much simpler than that in [11] and can easily converted into a linear time algorithm for the Abelian group isomorphism problem. The structure for proving our theorem for the sublinear time algorithm is shown in Figure 1.

An undirected graph $G=(V, E)$ consists a set of nodes $V$ and a set of undirected edges $E$ such that the two nodes of each edge in $E$ belong to set $V$. A path of $G$ is a series of nodes $v_{1} v_{2} \cdots v_{k}$ such that $\left(v_{i}, v_{i}+1\right)$ is an edge of $G$ for $i=1, \cdots, k-1$. A undirected graph is connected if every pair of nodes is linked by a path. A graph $G_{1}=\left(V_{1}, E_{1}\right)$ is a subgraph of $G=(V, E)$ if $E_{1} \subseteq E$ and $V_{1} \subseteq V$. A connected component of $G$ is a (maximal) subgraph $G_{1}=\left(V_{1}, E_{1}\right)$ of $G$ such that $G_{1}$ is a connected subgraph and $G$ does not have another connected subgraph $G_{2}=\left(V_{2}, E_{2}\right)$ with $E_{1} \subset E_{2}$ or $V_{1} \subset V_{2}$.

A directed graph $G=(V, E)$ consists of a set of nodes $V$ and a set of directed edges $E$ such that each edge in $E$ starts from one node in $V$ and ends at another node in $V$. A path of $G$ is a series of nodes 
$v_{1} v_{2} \cdots v_{k}$ such that $\left(v_{i}, v_{i}+1\right)$ is a directed edge of $G$ for $i=1, \cdots, k-1$. A (directed) cycle of $G$ is a directed path $v_{1} v_{2} \cdots v_{k}$ with $v_{1}=v_{k}$. For a directed graph $G=(V, E)$, let $G=\left(V, E^{\prime}\right)$ be the undirected graph that $E^{\prime}$ is derived from $E$ by converting each directed edge of $E$ into undirected edge. A directed graph $G=(V, E)$ is weakly connected if $G=\left(V, E^{\prime}\right)$ is connected. A subgraph $G_{1}=\left(V_{1}, E_{1}\right)$ of $G=(V, E)$ is a weakly connected component of $G$ if $\left(V_{1}, E_{1}^{\prime}\right)$ is a connected component of $\left(V, E^{\prime}\right)$.

We need the following lemma that shows the structure of a special kind directed graph in which each of its nodes has exactly one outgoing edge.

Lemma 15. Assume that $G=(E, V)$ is a weakly connected directed graph such that each node has exactly one outgoing edge that leaves it (and may come back to the node itself). Then the directed graph $G=(V, E)$ has the following properties: 1) Its derived undirected graph $G^{\prime}=\left(V, E^{\prime}\right)$ has exactly one cycle. 2) $G$ has exactly one directed cycle. 3)Every node of $G$ is either in the directed cycle or has a directed path to a node in the directed cycle. 4)For every node $v$ of $G$, if $v$ is not in the cycle of $G$, then there exists a node $v^{\prime}$ in the cycle of $G$ such that every path from $v$ to another node $v^{\prime \prime}$ in the cycle of $G$ must go through the node $v^{\prime}$.

Proof: $\quad$ Since each node of $G$ has exactly one edge leaving it, the number of edges in $G$ is the same as the number of nodes. Therefore, $G^{\prime}$ can be considered to be formed by adding one edge to a tree. Clearly, $G^{\prime}$ has exactly one cycle. Therefore, $G$ has at most one directed cycle.

Now we prove that $G$ have at least one directed cycle. We pick up a node from $G$. Since each node of $G$ has exactly one edge leaving it, follow the edge leaving the node to reach another node. We will eventually come back to the node that is visited before since $G$ has a finite number of nodes. Therefore, $G$ has at least one cycle. Therefore, $G$ has exactly one directed cycle. This process also shows that every node of $G$ has a directed path linking to a node in the directed cycle.

Assume that $v$ is a node of $G$ and $v$ is not in the cycle. Let $v^{\prime}$ be the first node such that $v$ has a path to $v^{\prime}$ and the path does not visit any other node in the cycle of $G$. Let $e$ be the edge leaving $v^{\prime}$. Clearly, $H=\left(V,(E-e)^{\prime}\right)$ is a tree. Therefore, for every node $v^{\prime \prime}$ in the cycle of $G$, every path in $(V, E-e)$ from $v$ to $v^{\prime \prime}$ has to go through $v^{\prime}$. It is still true when $e$ is added back since $e$ connects $v^{\prime}$.

Lemma 16. There exists an $O(n)$ time algorithm such that given an Abelian group $G$ of size $n$, prime $p \mid n$, and a table $H$ with $H(a)=a^{p}$, it returns two subgroups $G^{\prime}=\left\{a \in G \mid a^{p^{n_{1}}}=e\right\}$ and $G^{\prime \prime}=\left\{a^{p^{n_{1}}} \mid a \in G\right\}$ such that $\left|G^{\prime}\right|=p^{n_{1}},\left|G^{\prime \prime}\right|=m_{2}$ and $G=G^{\prime} \circ G^{\prime \prime}$, where $n=p^{n_{1}} m_{2}$ with $\left(p, m_{2}\right)=1$.

Proof: It is easy to see that $G^{\prime}$ can be derived in $O(n)$ time since we have the table $H$ available. By Lemma 2 , we have $G=G^{\prime} \circ G^{\prime \prime}$. We focus on how to generate $G^{\prime \prime}$ below. For each element $a$, set up a flag that is initially assigned -1 . In order to decompose the group $G$ into $G^{\prime} \circ G^{\prime \prime}$ with $\left|G^{\prime}\right|=p^{n_{1}}$ and $\left|G^{\prime \prime}\right|=m_{2}$, we use Lemma 2 to build up two subsets $A$ and $B$ of $G$, where $A=\left\{a \in G \mid a^{p^{n_{1}}}=e\right\}$ and $B=\left\{a^{p^{n_{1}}} \mid a \in G\right.$ and $\left.a^{p^{n_{1}}} \neq e\right\}$. Then let $G^{\prime}=A$ and $G^{\prime \prime}=B \cup\{e\}$.

During this construction, we have the table $H$ such that $H(a)=a^{p}$ for every $a \in G$. We compute $a^{p^{j}}$ for $j=1,2, \cdots, n_{1}$. If $a^{p^{j}}=e$ for some least $j$ with $1 \leq j \leq n_{1}$, put $a$ into $A$ and change the flag from -1 to 1 .

It is easy to see we can obtain all elements of $A$ in $O(n)$ steps. We design an algorithm to obtain $B$ by working on the elements in $G-A$. We build up some trees for the elements in $V_{0}=G-A$.

\section{Algorithm B}

Input:

group $G$, its size $n$ and $p$ with $p \mid n$;

table $H\left(\right.$ ) with $H(a)=a^{p}$ for each $a \in G$;

Output: subgroup $\left\{a^{p^{n_{1}}} \mid a \in G\right\}$;

begin

for every $a \in V_{0}$ with $a^{p}=b\left(\right.$ notice $\left.H(a)=a^{p}\right)$

begin

let $(a, b)$ be a directed edge from $a$ to $b$;

end (for)

form a directed graph $\left(V_{0}, E\right)$;

let $\left(E_{1}, V_{1}\right),\left(E_{2}, V_{2}\right), \cdots,\left(E_{m}, V_{m}\right)$ be the weakly connected components of $\left(E, V_{0}\right)$;

for each $\left(V_{i}, E_{i}\right)$ with $i=1,2, \cdots, m$ 
begin

find the loop $L_{i}$, and put all elements of the loop into the set $B$;

for each tree in $\left(V_{i}, E_{i}\right)-L_{i}$ compute the height of each node;

put all nodes of height at least $n_{1}$ into $B$;

end (for)

end

output $B$;

\section{End of Algorithm B}

For each component of $\left(E, V_{0}\right)$, each node has only one outgoing edge. It has at most one loop in the component (see Lemma 15 for the structure of such a directed graph). The height of a node in a subtree tree, which is derived from a weakly connected component by removing a directed cycle, is the length of longest path from a leaf to it. For each node $v$ in the cycle, clearly, there is a path $v_{0} v_{1} \cdots v_{n_{1}}$ with $v_{n_{1}}=v$ (notice that all the other nodes $v_{0}, v_{1}, \cdots, v_{n_{1}-1}$ are also in the cycle). Thus, $v \in B$. If $v$ is not in the cycle, $v \in B$ iff there is a path with length at least $n_{1}$ and the path ends $v$. Since each node has one outgoing edge, each node in the cycle has no edge going out the cycle. Thus, a node is in $B$ iff it has height of at least $n_{1}$ or it is in a cycle. Therefore, the set $B$ can be derived in $O(n)$ steps by using the depth first method to scan each tree.

Lemma 17. There is an $O(n)$ time algorithm such that given a group $G$ of size $n$, it returns the decomposition $G\left(p_{1}^{n_{1}}\right) \circ G\left(p_{2}^{n_{2}}\right) \circ \cdots \circ G\left(p_{t}^{n_{t}}\right)$, where $n$ has the factorization $n=p_{1}^{n_{1}} p_{2}^{n_{2}} \cdots p_{t}^{n_{t}}$ and $G\left(p_{i}^{n_{i}}\right)$ is the subgroup of size $p_{i}^{n_{i}}$ of $G$ for $i=1,2, \cdots, t$.

Proof: $\quad$ For $n=p_{1}^{n_{1}} p_{2}^{n_{2}} \cdots p_{t}^{n_{t}}$, assume that $p_{1}<p_{2}<\cdots<p_{t}$. We discuss the following two cases.

Case 1: $p_{1}>2$. In this case, 2 is the least prime that is not a divisor of $n$. By Theorem 14, we can find the order of all elements in $O(n \log p)=O(n)$ time since $p=2$ here. By Lemma 3, we can obtain the group decomposition in $O(n)$ time.

Case 2: $p_{1}=2$. Apply Lemma 16 , we have $G=G\left(2^{n_{1}}\right) \circ G^{\prime}$. In the next stage, we decompose $G^{\prime}$ into the production of subgroups $G^{\prime}=G\left(p_{2}^{n_{2}}\right) \circ \cdots \circ G\left(p_{t}^{n_{t}}\right)$. Since $G^{\prime}$ does not have the divisor 2, we come back to Case 1. Clearly, the total number of steps is $O(n)$.

Theorem 18. There is an $O(n)$ time algorithm for computing the basis of an Abelian group with $n$ elements.

Proof: The theorem follows from Lemma 17 and Lemma 12.

\section{Sublinear Time Algorithm for the Basis of Abelian Group}

In this section, we present a sublinear time algorithm for finding the basis of a finite Abelian group. For $n=p_{1}^{n_{1}} \cdots p_{k}^{n_{k}}$, we derive a randomized algorithm with $O\left(\left(\sum_{i=1}^{k} p_{i}^{n_{i}-1} n_{i}^{2} \log p_{i}\right)(\log n)(\log \log n)\right)$ running time (Theorem 21). For this sublinear time algorithm, we always assume that the Abelian group size $n$ and the prime factorization of $n$ are a part of the input. We give a self-contained proof for Theorem 21 that implies that the algorithm can find the basis for most Abelian groups in $(\log n)^{O(1)}$ time. The structure for proving Theorem 21 for the sublinear time algorithm is shown in Figure 2.

We also derive an $O\left(\left(\sum_{i=1}^{k} p_{i}^{n_{i} / 2} n_{i}^{2}\right)(\log n) \log \log n\right)$-time randomized algorithm to compute the basis of Abelian group $G$ of size $n$ (Theorem 28). Using a theorem of Buchmann and Schmidt [2], we obtain Theorem 28, which improves Theorem 21 and shows that there exists a sublinear time algorithm to compute the basis for every Abelian group. The structure for proving Theorem 28 for the sublinear time algorithm is shown in Figure 3.

We assume that $G$ has $n$ elements $a_{1}, \cdots, a_{n}$ and each $a_{i}$ is represented by an integer. The integer representation has the advantage in that those elements have linear order and we can use B-tree to store them so that finding and inserting can be done in $O(\log n)$ steps. We first present a sublinear time algorithm for computing the basis of a $G$ group, which has $p^{t}$ elements for some integer $t \geq 1$ and prime $p$.

\section{Algorithm C}




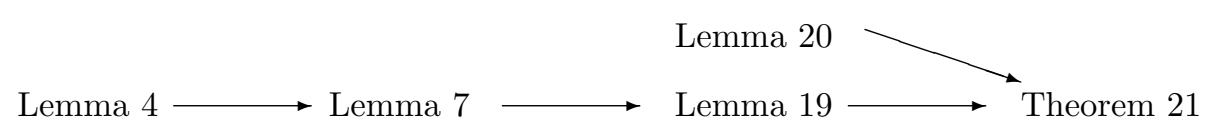

Figure 2: Structure for Proving Theorem 21 that implies most Abelian groups can be factorized in sublinear time

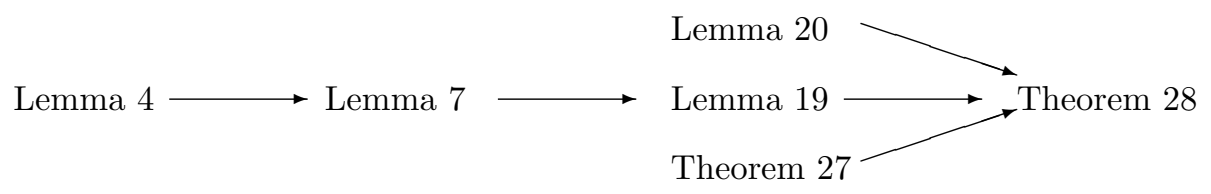

Figure 3: Structure for Proving Theorem 28 that implies every Abelian group can be factorized in sublinear time

Input: an Abelian group $G$, its size $p^{t}, p$ and $t$;

Output: a basis of $G$;

Phase 0:

If $t=0$ then output $e$ as the basis for $G$ and stop the algorithm;

Else

Let $H_{0}=\{e\}$;

Let $m=\left[\frac{x+\log t}{\log p}\right]$;

Enter Phase 1;

Phase 1:

Randomly select $m$ elements $b_{1}, \cdots, b_{m}$ from $G$.

Compute the orders of $b_{1}, \cdots, b_{m}$ (since each $\operatorname{ord}\left(b_{i}\right)=p^{\eta_{i}}$, we just save $\left.\left.\log _{p}\left(\operatorname{ord}\left(b_{i}\right)\right)=\eta_{i}\right)\right)$;

Let $a_{1}^{\prime}=b_{j}$, where $b_{j}$ has the largest order $\operatorname{ord}\left(b_{j}\right)=\max \left\{\operatorname{ord}\left(b_{i}\right)(i=1, \cdots, m)\right\}$;

Let $E_{1}=\log _{p}\left(\operatorname{ord}\left(a_{1}^{\prime}\right)\right)$;

If $\left(E_{1}<t\right)$ then

Begin

$H_{1}=\left\{a_{1}^{\prime j} \mid j=0,1, \cdots, \operatorname{ord}\left(a_{1}^{\prime}\right)-1\right\} ;$

Put all elements of $H_{1}$ in a B-tree;

Enter Phase 2;

End (then)

Else output $a_{1}^{\prime}$ as the basis of $G$ and stop the algorithm;

Phase $s+1$ :

Assume that $a_{1}^{\prime}, \cdots, a_{s}^{\prime}$ have been found at the Phases 1 to $s$;

Randomly select $m$ elements $b_{1}, \cdots, b_{m}$ from $G$.

For each $b_{i}((i=1,2, \cdots, m)$

Begin 
For each $g \in H_{s}$

Begin

Let $b=g b_{i}$;

Compute the order $p^{u}$ for $b$ and the set $B=\left\{b, b^{p}, b^{p^{2}}, \cdots, b^{p^{u-1}}\right\}$;

$\left(\right.$ Save $\left.\log _{p}(\operatorname{ord}(b))=u\right)$;

If $\left(\operatorname{ord}(b) \leq \operatorname{ord}\left(a_{s}^{\prime}\right)\right.$ and $\left.\left(B \cap H_{s}=\emptyset\right)\right)$ Then

Begin

Let $b_{i}^{\prime}=g b_{i}$;

Goto $L$;

End (if)

End (for)

L: Continue;

End (for)

Let $a_{s+1}^{\prime}=b_{j}^{\prime}$, where $b_{j}^{\prime}$ has the largest order ord $\left(b_{j}^{\prime}\right)=\max \left\{\operatorname{ord}\left(b_{i}^{\prime}\right)(i=1, \cdots, m)\right\}$;

Let $E_{s+1}=E_{s}+\log _{p}\left(\operatorname{ord}\left(a_{s+1}^{\prime}\right)\right)$;

If $\left(E_{s+1}<t\right)$ then

Begin

$H_{s+1}=\left\{a_{s+1}^{\prime j} h \mid h \in H_{s}\right.$ and $\left.j=0,1, \cdots, \operatorname{ord}\left(a_{s+1}^{\prime}\right)-1\right\} ;$

Put all elements of $H_{s+1}$ in a B-tree;

Enter the phase $s+2$;

End (Then)

Else output $a_{1}^{\prime}, a_{2}^{\prime}, \cdots, a_{s+1}^{\prime}$ as the basis for $G$ and halt the algorithm.

\section{End of Algorithm C}

For a finite set $A$, a random element $r$ of $A$ has the probability that with probability $\frac{1}{|A|}, r=a$ for every $a \in A$. The following fact is easy to verify by the definition of the basis and will be used in the proof of the algorithm.

Fact 1. Let $b_{1}, b_{2}, \cdots, b_{k}$ be the basis of Abelian group $G$. For each $i \in\{1,2, \cdots, k\}$, an random element $r$ is has the format $b_{i}^{\eta} c$, where $c$ is a random element in $\left\langle b_{1}, b_{2}, \cdots, b_{i-1}, b_{i+1}, \cdots, b_{k}\right\rangle$ and $\eta$ is a random number in $\left\{0,1, \cdots, \operatorname{ord}\left(b_{i}\right)-1\right\}$.

Lemma 19. (i) There exists a randomized algorithm such that given an Abelian group $G$ of size $p^{t}, p, t \geq 0$, and integer $x>0$, it runs in $O\left(p^{t-1}(x+\log t) t^{2} \log p\right)$ steps, uses at most $\left\lceil\frac{x+\log t}{\log p}\right\rceil t$ random elements selected from $G$, and computes its basis with a failure probability at most $\frac{1}{2^{x}}$.

(ii) With probability at most $\frac{1}{2^{x}}$, a set of $\left\lceil\frac{x+\log t}{\log p}\right\rceil t$ random elements of $G$ is not a set of generators of $G$.

Proof: Assume that $a_{1}, \cdots, a_{k}$ form a basis of $G$ with orders $\operatorname{ord}\left(a_{1}\right) \geq \operatorname{ord}\left(a_{2}\right) \geq \cdots \geq \operatorname{ord}\left(a_{k}\right)$. Our algorithm finds a basis $\left\{a_{1}^{\prime}, a_{2}^{\prime}, \cdots, a_{k}^{\prime}\right\}$ of $G$ with $\operatorname{ord}\left(a_{1}^{\prime}\right)=\operatorname{ord}\left(a_{1}\right), \operatorname{ord}\left(a_{2}^{\prime}\right)=\operatorname{ord}\left(a_{2}\right), \cdots$, and $\operatorname{ord}\left(a_{k}^{\prime}\right)=$ $\operatorname{ord}\left(a_{k}\right)$.

If $m \geq \frac{x+\log t}{\log p}$, then $m \log p \geq x+\log t$. It implies $\frac{1}{p^{m}} \leq \frac{1}{t 2^{x}}$. The algorithm is described in Algorithm C

Phase 0: If $t=0$ then output $e$ as the basis for $G$ and stop the algorithm. Otherwise, let $H_{0}=\{e\}$ and $m=\left\lceil\frac{x+\log t}{\log p}\right\rceil$. Then enter Phase 1. We set $m=\left\lceil\frac{x+\log t}{\log p}\right\rceil$ in the algorithm for the number of random sample elements selected from $G$ to find one element in the basis of $G$ in the coming phases.

Phase 1: Randomly select $m$ elements $b_{1}, \cdots, b_{m}$ from $G$. Assume that $b_{i}=a_{1}^{\eta_{i}} c_{i}$, where $c_{i} \in\left\langle a_{2}, \cdots, a_{k}\right\rangle$ and $\eta_{i}$ is an integer in the interval $\left[0, \operatorname{ord}\left(a_{1}\right)-1\right]$. If $\left(\eta_{i}, p\right)=1$, then $\operatorname{ord}\left(b_{i}\right)=\operatorname{ord}\left(a_{1}\right)$ (notice that $\operatorname{ord}\left(a_{1}\right)=p^{j}$ for some integer $j \geq 1$ and $\left.\operatorname{ord}\left(a_{1}\right) \geq \operatorname{ord}\left(a_{2}\right) \geq \cdots \geq \operatorname{ord}\left(a_{k}\right)\right)$. Since $b_{i}$ is a random element in $G, \eta_{i}$ is a random number in $\left[0, \operatorname{ord}\left(a_{1}\right)-1\right]$ and the probability is $\frac{1}{\operatorname{ord}\left(a_{1}\right)}$ that $\eta_{i}$ is equal to any integer in $\left[0, \operatorname{ord}\left(a_{1}\right)-1\right]$ (by Fact 1$)$. Assume that $\operatorname{ord}\left(a_{1}\right)=p^{j_{1}}$. There are $p^{j_{1}-1}$ integers $i$ in $\left[0, \operatorname{ord}\left(a_{1}\right)-1\right]$ with $(i, p) \neq 1$. With probability at most $\frac{p^{j_{1}-1}}{p^{j_{1}}}=\frac{1}{p},\left(\eta_{i}, p\right) \neq 1$. With probability at most $\frac{1}{p^{m}},\left(\eta_{i}, p\right) \neq 1$ for every $i=1, \cdots, m$. Therefore, with probability at most $\frac{1}{p^{m}}, \max \left\{\operatorname{ord}\left(b_{1}\right), \cdots, \operatorname{ord}\left(b_{m}\right)\right\}<\operatorname{ord}\left(a_{1}\right)$. 
Computing the orders of $b_{1}, \cdots, b_{m}$ takes $O\left(m \cdot t^{2} \log p\right)$ steps. This is because for each $b_{i}(i=1, \cdots, m)$, we compute $b_{i}^{p}, b_{i}^{p^{2}}, \cdots, b_{i}^{p^{t}}$ and take $O\left(\log p^{t}\right)$ steps for each of them. Let $a_{1}^{\prime}=b_{i}$ with $\operatorname{ord}\left(b_{i}\right)=\max \left\{\operatorname{ord}\left(b_{j}\right) \mid j=\right.$ $1, \cdots, m\}$. Clearly, with probability at most $\frac{1}{p^{m}}, \operatorname{ord}\left(a_{1}^{\prime}\right)=\operatorname{ord}\left(a_{1}\right)$ is not true. At the end of this phase, the algorithm checks if $G=\left\langle a_{1}^{\prime}\right\rangle$, which is equivalent to $\operatorname{ord}\left(a_{1}^{\prime}\right)=p^{t} \operatorname{or} \log _{p} \operatorname{ord}\left(a_{1}^{\prime}\right)=t$. If not, it generates $H_{1}=\left\langle a_{1}^{\prime}\right\rangle$ (all elements of $H_{1}$ are stored in a B-tree), and then enter Phase 2.

Phase $s+1$ : Assume that $a_{1}^{\prime}, \cdots, a_{s}^{\prime}$ have been obtained such that $\operatorname{ord}\left(a_{i}^{\prime}\right)=\operatorname{ord}\left(a_{i}\right)$ for $i=1, \cdots, s$, $a_{1}^{\prime}, \cdots, a_{s}^{\prime}$ are independent, and $H_{s}=\left\langle a_{1}^{\prime}, \cdots, a_{s}^{\prime}\right\rangle$. We will find $a_{s+1}^{\prime}$ in this phase. By Lemma 7 , there are $a_{s+1}^{\prime \prime}, \cdots, a_{k}^{\prime \prime}$ such that $a_{1}^{\prime}, \cdots, a_{s}^{\prime}, a_{s+1}^{\prime \prime}, \cdots, a_{k}^{\prime \prime}$ form the basis of $G$ with ord $\left(a_{1}^{\prime}\right)=\operatorname{ord}\left(a_{1}\right), \operatorname{ord}\left(a_{2}^{\prime}\right)=$ $\operatorname{ord}\left(a_{2}\right), \cdots, \operatorname{ord}\left(a_{s}^{\prime}\right)=\operatorname{ord}\left(a_{s}\right), \operatorname{ord}\left(a_{s+1}^{\prime \prime}\right)=\operatorname{ord}\left(a_{s+1}\right), \cdots$, and $\operatorname{ord}\left(a_{k}^{\prime \prime}\right)=\operatorname{ord}\left(a_{k}\right)$. If $b$ is a random element from $G$, then $b=a_{s+1}^{\prime \prime \eta} c$ and $\eta$ is a random integer in $\left[0, \operatorname{ord}\left(a_{s+1}^{\prime \prime}\right)-1\right]$ (by Fact 1$)$, where $c \in$ $\left\langle a_{1}^{\prime}, \cdots, a_{s}^{\prime}, a_{s+2}^{\prime \prime}, a_{s+3}^{\prime \prime}, \cdots, a_{k}^{\prime \prime}\right\rangle$.

Randomly select $m$ elements $b_{1}, \cdots, b_{m}$ from $G$. Let $b_{i}=a_{s+1}^{\prime \prime \eta_{i}} c_{i}$ and $\eta_{i}$ be a random integer in $\left[0, \operatorname{ord}\left(a_{s+1}^{\prime \prime}\right)-1\right]$, where $c_{i} \in\left\langle a_{1}^{\prime}, \cdots, a_{s}^{\prime}, a_{s+2}^{\prime \prime}, a_{s+3}^{\prime \prime}, \cdots, a_{k}^{\prime \prime}\right\rangle$. Similar to Phase 1 , the probability is at most $\frac{1}{p^{m}}$ that $\left(\eta_{i}, p\right) \neq 1$ for every $i=1, \cdots, m$.

For each $b_{u}$, we can always find another $g \in H_{s}$ such that $\operatorname{ord}\left(b_{u} g\right) \leq \operatorname{ord}\left(a_{s}^{\prime}\right)$ and $\left(\left\langle a_{1}^{\prime}, \cdots, a_{s}^{\prime}\right\rangle\right) \cap\left\langle b_{u} g\right\rangle=$ $\{e\}$. This is because we can let $g=a_{1}^{\prime-j_{1}} \cdots a_{s}^{\prime-j_{s}}$ when $b_{u}=a_{1}^{\prime j_{1}} \cdots a_{s}^{\prime j_{s}} a_{s+1}^{\prime \prime j_{s+1}} \cdots a_{k}^{\prime \prime j_{k}}$.

Assume that $g \in\left\langle a_{1}^{\prime}, \cdots, a_{s}^{\prime}\right\rangle$ and $\left\langle g b_{u}\right\rangle \cap H_{s}=\{e\}$. Let $g b_{u}=\prod_{i=1}^{s} a_{i}^{\prime t_{i} p^{\xi_{i}}} \prod_{j=s+1}^{k} a_{j}^{\prime \prime t_{j} p^{\xi_{j}}}$, where $\left(t_{i}, p\right)=1$ for $i=1, \cdots, k$. We claim that (a) $\max \left\{\operatorname{ord}\left(a_{i}^{\prime t_{i} p^{\xi_{i}}}\right) \mid i=1, \cdots, s\right\} \leq \max \left\{\operatorname{ord}\left(a_{j}^{\prime \prime t_{j}} p^{\xi_{j}}\right) \mid j=\right.$ $s+1, \cdots, k\}$; and $(\mathrm{b}) \operatorname{ord}\left(g b_{u}\right)=\max \left\{\operatorname{ord}\left(a_{j}^{\prime \prime t_{j} p^{\xi_{j}}}\right) \mid j=s+1, \cdots, k\right\}$. Assume (a) is not true. We have that $\max \left\{\operatorname{ord}\left(a_{i}^{\prime t_{i} p^{\xi_{i}}}\right) \mid i=1, \cdots, s\right\}>\max \left\{\operatorname{ord}\left(a_{j}^{\prime \prime t_{j} p^{\xi_{j}}}\right) \mid j=s+1, \cdots, k\right\}$. Let $\max \left\{\operatorname{ord}\left(a_{j}^{\prime \prime t_{j} p^{\xi_{j}}}\right) \mid j=s+1, \cdots, k\right\}=$ $p^{\xi}$. Then $\left(\prod_{j=s+1}^{s} a_{j}^{\prime \prime t_{j} p^{\xi_{j}}}\right)^{p^{\xi}}=e$ and $\left(\prod_{i=1}^{s} a_{i}^{\prime t_{i} p^{\xi_{i}}}\right)^{p^{\xi}} \neq e$. Thus, $e \neq\left(g b_{u}\right)^{p^{\xi}} \in H_{s}\left(\right.$ recall $\left.H_{s}=\left\langle a_{1}, \cdots, a_{s}\right\rangle\right)$. This contradicts that $\left\langle g b_{u}\right\rangle \cap H_{s}=\{e\}$. Therefore, (a) is true. (b) follows from (a).

If $b_{i}=a_{s+1}^{\prime \prime \eta_{i}} c_{i}$ with $c_{i} \in\left\langle a_{1}^{\prime}, \cdots, a_{s}^{\prime}, a_{s+2}^{\prime \prime}, a_{s+3}^{\prime \prime}, \cdots, a_{k}^{\prime \prime}\right\rangle$. We find $b_{i}^{\prime}=g b_{i} \operatorname{such}$ that $\operatorname{ord}\left(g b_{i}\right) \leq \operatorname{ord}\left(a_{s}^{\prime}\right)$ and $\left\langle g b_{i}\right\rangle \cap H_{s}=\{e\}$. The $s+1$-th element $a_{s+1}^{\prime}$ is selected to be $b_{j}^{\prime}$ with $\operatorname{ord}\left(b_{j}^{\prime}\right)=\max \left\{\operatorname{ord}\left(b_{i}^{\prime}\right)(i=1, \cdots, m)\right\}$. If $\left(\eta_{i}, p\right)=1$, then $\operatorname{ord}\left(b_{i}^{\prime}\right)=\operatorname{ord}\left(a_{s+1}^{\prime \prime}\right)=\operatorname{ord}\left(a_{s+1}\right)$. We already know that the probability is at most $\frac{1}{p^{m}}$ that $\left(\eta_{i}, p\right) \neq 1$ for every $i=1, \cdots, m$. Thus, with probability at most $\frac{1}{p^{m}}, \operatorname{ord}\left(a_{s+1}^{\prime}\right) \neq \operatorname{ord}\left(a_{s+1}\right)$. At the end of this phase, the algorithm checks if $G=\left\langle a_{1}^{\prime}, a_{2}^{\prime}, \cdots, a_{s}^{\prime}, a_{s+1}^{\prime}\right\rangle$, which is equivalent to that $\log _{p}\left(\operatorname{ord}\left(a_{1}^{\prime}\right)\right)+\log _{p}\left(\operatorname{ord}\left(a_{2}^{\prime}\right)\right)+\cdots+\log _{p}\left(\operatorname{ord}\left(a_{s+1}^{\prime}\right)\right)=t$. If not, it generates $H_{s+1}=H_{s} \circ\left\langle a_{s+1}^{\prime}\right\rangle=$ $\left\langle a_{1}^{\prime}, \cdots, a_{s}^{\prime}, a_{s+1}^{\prime}\right\rangle$ (all elements of $H_{s+1}$ are stored in a B-tree) and enter Phase $s+2$.

Assume the algorithm stops at Phase $z+1$. The basis generated by the algorithm is $a_{1}^{\prime}, \cdots, a_{z}^{\prime}, a_{z+1}^{\prime}$. Thus, $H_{1}, H_{2}, \cdots, H_{z}$ have been generated with $H_{1} \subset H_{2} \subset \cdots \subset H_{z} \subset G$. The size of $H_{z}$ is strictly less than that of the group $G$. It is easy to see that $H_{i}=\left\langle a_{1}^{\prime}, a_{2}^{\prime}, \cdots, a_{i}^{\prime}\right\rangle$, which is the subgroup generated by the part of elements that have been found from Phase 1 to Phase $i$ for $(i=1, \cdots, z)$. Thus, $\left|H_{i}\right|=\prod_{j=1}^{i} \operatorname{ord}\left(a_{j}^{\prime}\right)$. The algorithm stops at Phase $z+1$, which has found the full basis $a_{1}^{\prime}, \cdots, a_{z+1}^{\prime}$ and it does not generate $H_{z+1}$ any more. This is why we use less than than linear time. It is easy to see that $\left|H_{z}\right| \leq p^{t-1}$ and $\left|H_{y-1}\right| \leq \frac{\left|H_{y}\right|}{p}$ for $y=z, z-1, \cdots, 2,1$. We have $\left|H_{z}\right|+\left|H_{z-1}\right|+\cdots+\left|H_{1}\right|=O\left(p^{t-1}+\cdots+p^{2}+p\right)=O\left(\frac{p^{t}-1}{p-1}\right)=O\left(p^{t-1}\right)$.

For each $b_{i}$, it takes $\left|H_{s}\right|$ steps to generate all $b=g b_{i}$ for all $g \in H_{s}$. For each $b=g b_{i}$, it takes $O\left(t \log p^{t}\right)=O\left(t^{2} \log p\right)$ steps to compute its order ord $(b)=p^{u}$ and the set $B=\left\{b, b^{p}, b^{p^{2}}, \cdots, b^{p^{u-1}}\right\}$ in the algorithm. It is easy to see that $H_{s} \cap\langle b\rangle=\{e\}$ if and only if $H_{s} \cap B=\emptyset$. It takes another $O\left(t \log p^{t}\right)=O\left(t^{2} \log p\right)$ steps for checking if $B \cap H_{s}=\emptyset$, which needs to use at most $t$ finding operations to a B-tree with at most $p^{t}$ elements. It takes $O\left(\left|H_{s}\right| t^{2} \log p\right)$ steps to compute one $b_{i}^{\prime}$. Thus, it takes $O\left(\left|H_{s}\right| m t^{2} \log p\right)$ steps to compute all $b_{i}^{\prime}$ s for $i=1,2, \cdots, m$.

The total running time is $\left.O\left(\left|H_{1}\right|+\cdots+\mid H_{z}\right) m t^{2} \log p\right)=O\left(\frac{p^{t-1}(x+\log t) t^{2} \log p}{\log p}\right)=O\left(p^{t-1}(x+\log t) t^{2} \log p\right)$. With probability at most $\frac{1}{p^{m}}$, one phase fails. There are at most $t$ phases since each phase generates a new element in the basis and the group has size $p^{t}$. The total probability of failure is at most $\frac{t}{p^{m}} \leq 2^{x}$ by the setting of $m$.

(ii)Let $B_{i}=\left\{b_{1}, b_{2}, \cdots, b_{m}\right\}$ be the set of randomized elements of $G$. Assume that (i) terminates at Stage $t$ and returns the basis $a_{1}^{\prime}, \cdots, a_{t}^{\prime}$. According to the proof of (i), we have that $a_{i}^{\prime}$ is generated by the elements in $\cup_{i=1}^{t} B_{i}$. Therefore, $\cup_{i=1}^{t} B_{i}$ is a set of generators of $G$. The number of elements in $\cup_{i=1}^{t} B_{i}$ is bounded by 
$\left\lceil\frac{x+\log t}{\log p}\right\rceil t$. The failure probability is at most that of (i).

Lemma 20. Let $n=p_{1}^{n_{1}} \cdots p_{k}^{n_{k}}$ and $G$ be an Abelian group of $n$ elements. Assume $m_{i}=\frac{n}{p_{i}^{n_{i}}}$ for $i=1, \cdots, k$. If a is a random element of $G$ that with probability $\frac{1}{|G|}$, a is equal to $b$ for each $b \in G$, then $a^{m_{i}}$ is a random element of $G\left(p_{i}^{n_{i}}\right)$, the subgroup of $G$ with $p^{n_{i}}$ elements, such that with probability $\frac{1}{p_{i}^{n_{i}}}, a^{m_{i}}$ is $b$ for any $b \in G\left(p_{i}^{n_{i}}\right)$

Proof: $\quad$ Let $b_{i, j}\left(j=1, \cdots, k_{i}\right)$ be the basis of $G\left(p_{i}^{n_{i}}\right)$, i.e. $G\left(p_{i}^{n_{i}}\right)=\left\langle b_{i, 1}\right\rangle \circ \cdots \circ\left\langle b_{i, k}\right\rangle$. Assume $a$ is a random element in $G$. Let $a=\left(\prod_{j=1}^{k_{i}} b_{i, j}^{c_{i, j}}\right) a^{\prime}$, where $a^{\prime}$ is an element in $\prod_{j \neq i} G\left(p_{j}^{n_{j}}\right)$. For every two integers $x \neq y \in\left[0, p_{i}^{n_{i}}-1\right], m_{i} x \neq m_{i} y\left(\bmod p_{i}^{n_{i}}\right)$ (Otherwise, $m_{i} x=m_{i} y\left(\bmod p_{i}^{n_{i}}\right)$ implies $x=y$ because $\left.\left(m_{i}, p_{i}\right)=1\right)$. Thus, the list of numbers $m_{i} \cdot 0\left(\bmod o\left(p_{i}^{t}\right)\right), m_{i} \cdot 1\left(\bmod o\left(p_{i}^{t}\right)\right), \cdots, m_{i}\left(p_{i}^{t}-1\right)\left(\bmod o\left(p_{i}^{t}\right)\right)$ is a permutation of $0,1, \cdots, p_{i}^{t}-1$. Thus, if $c_{i, j}$ is a random integer in the range $\left[0, \operatorname{ord}\left(b_{i, j}\right)-1\right]$ such that with probability $\frac{1}{\operatorname{ord}\left(b_{i, j}\right)}, c_{i, j}=c^{\prime}$ for each $c^{\prime} \in\left[0, \operatorname{ord}\left(b_{i, j}\right)-1\right]$, then the probability is also $\frac{1}{\operatorname{ord}\left(b_{i, j}\right)} \operatorname{that}$ $m_{i} c_{i, j}=c^{\prime}$ for each $c^{\prime} \in\left[0, \operatorname{ord}\left(b_{i, j}\right)-1\right]$. Therefore, $a^{m_{i}}=\left(\left(\prod_{j=1}^{k_{i}} b_{i, j}^{c_{i, j}}\right) a^{\prime}\right)^{m_{i}}=\prod_{j=1}^{k_{i}} b_{i, j}^{m_{i} c_{i, j}}$, which is a random element in $G\left(p_{i}^{n_{i}}\right)$.

Theorem 21. There exists a randomized algorithm such that given an Abelian group $G$ of size $n$ with $n=p_{1}^{n_{1}} \cdots p_{k}^{n_{k}}$, the algorithm computes the basis of $G$ in $O\left(\left(\sum_{i=1}^{k} p_{i}^{n_{i}-1} n_{i}^{2} \log p_{i}\right)(\log n) \log \log n\right)$ running time.

Proof: Let $x=3 \log \log n$. Then $\frac{1}{2^{x}}<\frac{1}{(\log n)^{2}}$. It takes $O(\log n)$ steps for computing $a^{m_{i}}$ for an element $a \in G$, where $m_{i}=\frac{n}{p_{i}^{n_{i}}}$. Each random element of $G$ can be converted into a random element of $G\left(p_{i}^{n_{i}}\right)$ by Lemma 20. Each $G\left(p_{i}^{n_{i}}\right)$ needs $O\left(x+\log n_{i}\right) n_{i}$ random elements by Lemma 19. Each $G\left(p_{i}^{n_{i}}\right)$ needs $O\left(\left(x+\log n_{i}\right) n_{i} \log n\right)$ time to convert the $\left(x+\log n_{i}\right) n_{i}$ random elements from $G$ to $G\left(p_{i}^{n_{i}}\right)$. It takes $\left.O\left(\sum_{i=1}^{k}\left(x+\log n_{i}\right) n_{i} \log n\right)\right)$ time to convert random elements of $G$ into the random elements in all subgroups $G\left(p_{i}^{n_{i}}\right)$ for $i=1, \cdots, k$. For $n=p_{1}^{n_{1}} \cdots p_{k}^{n_{k}}, \sum_{i=1}^{k} n_{i} \log p_{i}=\log n$. Furthermore, $x+\log n_{i}=O(\log \log n)$. By Lemma 19, the sum of time for all $G\left(p_{i}^{n_{i}}\right)$ s to find basis is $O\left(\left(\sum_{i=1}^{k} p_{i}^{n_{i}-1}\left(\log \log n+\log n_{i}\right) n_{i}^{2} \log p_{i}\right)(\log n)\right)=$ $O\left(\left(\sum_{i=1}^{k} p_{i}^{n_{i}-1} n_{i}^{2} \log p_{i}\right)(\log n) \log \log n\right)$. This follows from Lemma 20 and Lemma 19. The group size $n$ has at most $\log n$ prime factors. Since each $G\left(p_{i}^{n_{i}}\right)$ has a probability at most $\frac{1}{2^{x}}$ of failure, the total probability of failure is at most $\frac{\log n}{2^{x}} \leq \frac{1}{\log n}$.

Corollary 22. There exists a randomized algorithm such that given an Abelian group $G$ of size $n$ with $n=p_{1}^{n_{1}} \cdots p_{k}^{n_{k}}$, the algorithm computes the basis of $G$ in $O\left(\min \left(n,\left(\sum_{i=1}^{k} p_{i}^{n_{i}-1} n_{i}^{2} \log p_{i}\right)(\log n) \log \log n\right)\right)$ running time.

Proof: Assume that $n=p_{1}^{n_{1}} \cdots p_{k}^{n_{k}}$ is the size of an Abelian group. If $n \geq\left(\sum_{i=1}^{k} p_{i}^{n_{i}-1} n_{i}^{2} \log p_{i}\right)(\log n) \log \log n$, then apply Theorem 18. Otherwise, apply Theorem 21.

Corollary 23. There exists a randomized algorithm with $O\left(\min \left(n, n^{1-\frac{1}{d}}(\log n)^{3}(\log \log n)\right)\right)$ running time that given an Abelian group $G$ of size $n=p_{1}^{n_{1}} \cdots p_{k}^{n_{k}}$, it computes the basis of $G$, where $d=\max \left\{n_{i} \mid i=\right.$ $1, \cdots, k\}$.

Proof: $\quad$ For $n=p_{1}^{n_{1}} \cdots p_{k}^{n_{k}}, \sum_{i=1}^{k} n_{i} \log p_{i}=\log n$ and $p_{i}^{n_{i}-1} \leq n^{1-\frac{1}{d}}$. If $n \geq n^{1-\frac{1}{d}}(\log n)^{3}(\log \log n)$, then by Theorem 21, the running time is $O\left(\left(\sum_{i=1}^{k} p_{i}^{n_{i}-1} n_{i}^{2} \log p_{i}\right)(\log n) \log \log n\right)=O\left(n^{1-\frac{1}{d}}(\log n)^{3}(\log \log n)\right)$. Otherwise, apply Theorem 21.

Definition 24. For an integer $n$, define $F(n)=\max \left\{p^{i-1}\left|p^{i}\right| n, p^{i+1} \not \nmid n, i \geq 1\right.$, and $p$ is a prime $\}$. Define $G(m, c)$ as the set of all integers in $[1, m]$ with $F(n) \geq(\log n)^{c}$ and $H(m, c)=|G(m, c)|$.

Theorem 25. $\frac{H(m, c)}{m}=O\left(\frac{1}{(\log m)^{c / 2}}\right)$ for every constant $c>0$. 
Proof: $\quad H(m, c)$ is the number of integers in $G(m, c)$, which is a subset of integers in $[1, m]$. We discuss the three cases.

The number of integers in the interval $\left[1, \frac{m}{(\log m)^{c / 2}}\right]$ is at most $\frac{m}{(\log m)^{c / 2}}$. We only consider those numbers in the range $I=\left[\frac{m}{(\log m)^{c / 2}}, m\right]$. It is easy to see that for every integer $n \in I, 2(\log n)^{c} \geq(\log m)^{c}$ for all large $m$ since $c$ is fixed. We consider each number $n \in I$ such that $p^{t} \mid n$ with $p^{t} \geq \frac{(\log m)^{c}}{2}$ for some prime $p$.

For each prime number $p \in\left[2,(\log m)^{c / 2}\right]$, let $t$ be the least integer with $p^{t} \geq \frac{(\log m)^{c}}{2}$. We count the number of integers $n \in I$ such that $p^{u} \mid n$ for some $u \geq t$. The number is at most $\frac{m}{p^{t}}+\frac{m}{p^{t+1}}+\cdots \leq$ $\frac{m}{p^{t}}\left(1+\frac{1}{2}+\frac{1}{2^{2}}+\cdots\right) \leq \frac{2 m}{p^{t}} \leq \frac{4 m}{(\log m)^{c}}$. Therefore, it has at most $(\log m)^{c / 2} \cdot \frac{2 m}{(\log m)^{c}} \leq \frac{4 m}{(\log m)^{c / 2}}$ integers $n \in I$ to have $p^{t} \mid n$ with $p^{t} \geq \frac{(\log m)^{c}}{2}$.

Let's consider the cases $p^{t} \mid n$ for $p>(\log m)^{c / 2}$ and $t \geq 2$. The number of integers $n \in I$ for a fixed $p$ with $p^{2} \mid n$ is at most $\frac{m}{p^{2}}+\frac{m}{p^{3}}+\cdots \leq \frac{2 m}{p^{2}}$. The total number of integers $n \in I$ that have $p^{2} \mid n$ for some prime number $p>(\log m)^{c / 2}$ is at most $\frac{2 m}{\left(1+(\log m)^{c / 2}\right)^{2}}+\frac{2 m}{\left(2+(\log m)^{c / 2}\right)^{2}}+\cdots<\frac{2 m}{\left((\log m)^{c / 2}\right)\left(1+(\log m)^{c / 2}\right)}+$ $\frac{2 m}{\left(\left(1+(\log m)^{c / 2}\right)\left(2+(\log m)^{c / 2}\right)^{2}\right.}+\cdots \leq \frac{2 m}{(\log m)^{c / 2}}$. Combining the cases above, we have $\frac{H(m, c)}{m}=O\left(\frac{1}{(\log m)^{c / 2}}\right)$.

Theorem 21 and Theorem 25 imply the following theorem:

Theorem 26. There exists a randomized algorithm such that if $n$ is in $[1, m]-G(m, c)$, then the basis of an Abelian group of size $n$ whose prime factorization is also part of the input can be computed in $O\left((\log n)^{c+3} \log \log n\right)$-time, where $c$ is an arbitrary positive constant and $G(m, c)$ is a subset of integers in $[1, m]$ with $\frac{|G(m, c)|}{m}=O\left(\frac{1}{(\log m)^{c / 2}}\right)$ for each integer $m$.

We apply a theorem of Buchmann and Schmidt [2] to improve our sublinear time algorithm for finding the basis of Abelian group. The improved algorithm is sublinear in all cases.

Theorem 27 ([2]). There exists an $O(m \sqrt{|G|})$ time algorithm such that given a set of generators of size $m$ for an Abelian group $G$, the algorithm returns the basis of $G$ in $O(m \sqrt{|G|})$ steps.

Theorem 28. There exists a randomized algorithm such that given an Abelian group $G$ of size $n$ with $n=p_{1}^{n_{1}} \cdots p_{k}^{n_{k}}$, the algorithm computes the basis of $G$ in $O\left(\left(\sum_{i=1}^{k} p_{i}^{n_{i} / 2} n_{i}^{2}\right)(\log n) \log \log n\right)$ running time.

Proof: $\quad$ Let $x=3 \log \log n$. Then $\frac{1}{2^{x}}<\frac{1}{(\log n)^{2}}$. It takes $O(\log n)$ steps to compute $a^{m_{i}}$ for an element $a \in G$, where $m_{i}=\frac{n}{p_{i}^{n_{i}}}$. Each random element of $G$ can be converted into a random element of $G\left(p_{i}^{n_{i}}\right)$ by Lemma 20. Each $G\left(p_{i}^{n_{i}}\right)$ needs $O\left(x+\log n_{i}\right) n_{i}$ random elements by Lemma 19 . Each $G\left(p_{i}^{n_{i}}\right)$ needs $O\left(\left(x+\log n_{i}\right) n_{i} \log n\right)$ time to convert the $\left(x+\log n_{i}\right) n_{i}$ random elements from $G$ to $G\left(p_{i}^{n_{i}}\right)$. It takes $\left.O\left(\sum_{i=1}^{k}\left(x+\log n_{i}\right) n_{i} \log n\right)\right)$ time to convert random elements of $G$ into the random elements in all subgroups $G\left(p_{i}^{n_{i}}\right)$ for $i=1, \cdots, k$. For $n=p_{1}^{n_{1}} \cdots p_{k}^{n_{k}}, \sum_{i=1}^{k} n_{i} \log p_{i}=\log n$. Furthermore, $x+\log n_{i}=O(\log \log n)$. By Theorem 27, the sum of time for all $G\left(p_{i}^{n_{i}}\right)$ s to find basis is $O\left(\left(\sum_{i=1}^{k} p_{i}^{n_{i} / 2}\left(\log \log n+\log n_{i}\right) n_{i}^{2}\right)(\log n)\right)=$ $O\left(\left(\sum_{i=1}^{k} p_{i}^{n_{i} / 2} n_{i}^{2}\right)(\log n) \log \log n\right)$

It follows from Lemma 20 and Lemma 19. The group size $n$ has at most $\log n$ prime factors. Since each $G\left(p_{i}^{n_{i}}\right)$ has a probability at most $\frac{1}{2^{x}}$ of failure, the total probability of failure is at $\operatorname{most} \frac{\log n}{2^{x}} \leq \frac{1}{\log n}$.

Theorem 28 and Theorem 25 imply the following theorem:

Theorem 29. There exists a randomized algorithm such that if $n$ is in $[1, m]-G(m, c)$, then the basis of an Abelian group of size $n$ whose prime factorization is also part of the input can be computed in $O\left((\log n)^{\frac{c}{2}+3} \log \log n\right)$-time, where $c$ is an arbitrary positive constant and $G(m, c)$ is a subset of integers in $[1, m]$ with $\frac{|G(m, c)|}{m}=O\left(\frac{1}{(\log m)^{c / 2}}\right)$ for each integer $m$. 


\section{Conclusion}

In this paper, we obtained an $O(n)$-time deterministic algorithm for computing the basis of an Abelian group with $n$ elements. We also derive an $O\left(n^{1 / 2}(\log n)^{3} \log \log n\right)$-time randomized algorithm to compute the basis of an Abelian group $G$ of size $n$. We also show that for each integer $n \in[1, m]-G(m, c)$, the basis of an Abelian group of size $n$ can be computed in $(\log n)^{\frac{c}{2}+3} \log \log n$ time $\left(n=p_{1}^{n_{1}} \cdots p_{t}^{n_{t}}\right.$ is the part of the input of the algorithm), where $c$ is a constant and $m$ is arbitrary integer. An interesting problem of further research is to obtain time lower bound to compute the basis of an Abelian group of $n$ elements.

\section{Acknoledgements}

We would like to thank Eric Allender and Igor Shparlinski for their help and comments on an earlier version of this paper.

\section{References}

[1] J. Buchman, M. J. Jacobson Jr., and E. Teske. On some computational problems in finite abelian groups. Mathematics of Computation, 66:1663-1687, 1997.

[2] J. Buchmann and A. Schmidt. Computing the structure of a finite abelian group. Mathematics of Computation, 74:2017-2026, 2005.

[3] L. Chen. Algorithms and their complexity analysis for some problems in finite group. Journal of Sandong Normal University, in Chinese, 2:27-33, 1984.

[4] K. Cheung and M. Mosca. Decomposing finite abelian groups. Quantum Information and Computation, $1: 26-32,2001$

[5] T. H. Cormen, C. E. Leiserson, R. L. Rivest, and C. Stein. Introduction to Algorithms, Second Edition. The MIT Press, 2001.

[6] Y. G. Desmedt and Y. Frankel. Homomorphic zero-knowledge threshold schemes over any finite abelian group. SIAM Journal on Discrete Mathematics, 7(4):667-679, 1994.

[7] M. Garzon and Y. Zalcstein. On isomorphism testing of a class of 2-nilpoten groups. Journal of Computer and System Sciences, 42:237-248, 1991.

[8] C. M. Hoffmann. Group-Theoretic Algorithms and Graph Isomorphism. Springer-Verlag, 1982.

[9] T. Hungerford. Algebra. Springer-Verlag, 1974.

[10] M. I. Kargapolov and J. I. Merzljako. Fundamentals of the Theory of Groups. Springer-Verlag, 1979.

[11] T. Kavitha. Linear time algorithms for abelian group isomorphism and related problem. Journal of Computer and System Sciences, To appear.

[12] T. Kavitha. Efficient algorithms for abelian group isomorphism and related problems. In Proceedings of Foundations of Software Technology and Theoretical Computer Science, Lecture notes in computer science, 2914, pages 277-288, 2003.

[13] A. Y. Kitaev. Quantum computations: Algorithms and error correction. Russian Math. Surveys, $52: 1191,1997$.

[14] J. Köbler, U. Schöning, and J. Toran. The Graph Isomorphism Problem: Its Structural Complexity. Birkhouser, 1993.

[15] C. Lomont. The hidden subgroup problem -review and open problems. http://arxiv.org/abs/quant$\mathrm{ph} / 0411037,2004$. 
[16] A. Menezes. Elliptic curve cryptosystems. CryptoBytes, 1:1-4, 1995.

[17] G. L. Miller. On the $n^{\log n}$ isomorphism technique. In Proceedings of the tenth annual ACM symposium on theory of computing, pages 128-142, 1978.

[18] G. L. Miller. Graph isomorphism, general remarks. Journal of Computer and System Sciences, 18:128$142,1979$.

[19] V. Miller. Uses of elliptic curves in cryptography. In Advances in Cryptology CRYPTO'85, Lecture Notes in Computer Science, pages 417-426, 1986.

[20] C. Savage. An $O\left(n^{2}\right)$ algorithm for abelian group isomorphism. Technical report, North Carolina State University, January 1980.

[21] D. Shanks. Class number, a theory of factorization and genera. Proc. Symp. Pure Math., 20:414-440, 1971.

[22] P. W. Shor. Polynomial-time algorithms for prime factorization and discrete logarithms on a quantum computer. SIAM Journal on Computing, 26:14841509, 1997.

[23] D. R. Simon. On the power of quantum computation. SIAM Journal on Computing, 26:1474 1483, 1997.

[24] N. Vikas. An $O(n)$ algorithm for abelian $p$-group isomorphism and an $O(n \log n)$ algorithm for abelian group isomorphism. Journal of Computer and System Sciences, 53:1-9, 1996. 